\title{
A Comparison of the Sherali-Adams, Lovász-Schrijver and Lasserre Relaxations for $0-1$ Programming
}

\author{
Monique Laurent \\ CWI, Kruislaan 413, 1098 SJ Amsterdam, The Netherlands
}

\begin{abstract}
Sherali and Adams (1990), Lovász and Schrijver (1991) and, recently, Lasserre (2001) have constructed hierarchies of successive linear or semidefinite relaxations of a $0-1$ polytope $P \subseteq \mathbb{R}^{n}$ converging to $P$ in $n$ steps. Lasserre's approach uses results about representations of positive polynomials as sums of squares and the dual theory of moments. We present the three methods in a common elementary framework and show that the Lasserre construction provides the tightest relaxations of $P$. As an application this gives a direct simple proof for the convergence of the Lasserre's hierarchy. We describe applications to the stable set polytope and to the cut polytope.
\end{abstract}

Keywords: $0-1$ polytope, linear relaxation, semidefinite relaxation, lift-and-project, stable set polytope, cut polytope.

\section{Introduction}

Given a set $F \subseteq\{0,1\}^{n}$, we are interested in finding the linear inequality description for the polytope $P:=\operatorname{conv}(F)$. A first (easy) step is to find a linear programming formulation for $P$; that is, to find a linear system $A x \leq b$ for which the polytope

$$
K:=\left\{x \in \mathbb{R}^{n} \mid A x \leq b\right\}
$$

satisfies $K \cap\{0,1\}^{n}=F$.

If all vertices of $K$ are integral then $K=\operatorname{conv}(F)$ and we are done. Otherwise we have to find 'cutting planes' permitting to strengthen the relaxation $K$ and to cut off its fractional vertices. Such cutting planes can be found by exploiting the combinatorial structure of the problem at hand. Extensive research has been done for finding (partial) linear descriptions for many polyhedra arising from specific combinatorial optimization problems. Next to that, research has also focused on developing general purpose methods applying to arbitrary $0-1$ problems or, more generally, integer programming problems.

One of the first such methods, which applies more generally to integral polyhedra, is the method of Gomory for generating cuts tightening the linear relaxation $K$. Given a linear inequality $\sum_{i} a_{i} x_{i} \leq \alpha$ valid for $K$ where all the coefficients $a_{i}$ are integers, the inequality $\sum_{i} a_{i} x_{i} \leq\lfloor\alpha\rfloor$ (known as a Gomory-Chvátal cut) is still valid for $\operatorname{conv}(F)$ but may eliminate some part of $K$. If we apply this transformation to any inequality $\sum_{i} a_{i} x_{i} \leq \alpha$ which can be obtained by taking linear combinations of the inequalities defining $K$ with suitable nonnegative multipliers ensuring that the $a_{i}$ 's are integral, then we obtain a polytope $K^{\prime}$ satisfying

$$
\operatorname{conv}(F) \subseteq K^{\prime} \subseteq K
$$


Set $K^{(1)}:=K^{\prime}$ and define recursively $K^{(t+1)}:=\left(K^{(t)}\right)^{\prime}$. Chvátal $(1973)$ proved that $K^{(t)}=\operatorname{conv}(F)$ for some $t$; the smallest $t$ for which this is true is the Chvátal rank of the polytope $K$. The Chvátal rank may be very large as it depends in general not only on the dimension $n$ but also on the coefficients of the inequalities involved. However, when $K$ is assumed to be contained in the cube $[0,1]^{n}$ then its Chvátal rank is bounded by $O\left(n^{2} \log n\right)$ (Eisenbrand and Schulz 1999). Even if we can optimize a linear objective function over $K$ in polynomial time optimizing a linear objective function over the first Chvátal closure $K^{\prime}$ is a co-NP-hard problem in general (Eisenbrand 1999).

Another popular method is to try to represent $P$ as the projection of another polytope $Q$ lying in a higher (but preferably still polynomial) dimensional space. The idea behind being that the projection of a polytope $Q$ may have more facets than $Q$ itself. Hence it could be that even if $P$ has an exponential number of facets, such $Q$ exists having only a polynomial number of facets and lying in a space whose dimension is polynomial in the original dimension of $P$ ( $\operatorname{such} Q$ is sometimes called a compact representation of $P$ ). If this is the case then we have a proof that any linear optimization problem over $P$ can be solved in polynomial time.

Several methods have been developed for constructing projection representations for general $0-1$ polyhedra; in particular, by Sherali and Adams (1990), by Lovász and Schrijver (1991), by Balas, Ceria and Cornuéjols (1993) and, recently, by Lasserre (2000,2001). A common feature of these methods is the construction of a hierarchy $K \supseteq K^{1} \supseteq K^{2} \supseteq \ldots \supseteq P$ of relaxations of $P$ which finds the exact convex hull in $n$ steps; that is, $K^{n}=P$. These relaxations are linear or semidefinite (in the case of Lovász-Schrijver and Lasserre). Moreover, under some assumptions over $K$, one can optimize in polynomial time a linear objective over an iterate $K^{t}$ for any fixed $t$.

The following inclusions are known among these various hierarchies: the Sherali-Adams iterate is contained in the Lovász-Schrijver iterate which in turn is contained in the Balas-Ceria-Cornuéjols iterate. The latter inclusion is an easy verification and the former was mentioned in (Lovász and Schrijver 1991) as an application of somewhat complicated algebraic manipulations; we present in Section 4 a simple direct proof for this inclusion.

The construction of Lasserre is motivated by results about representations of nonnegative polynomials as sums of squares and the dual theory of moments, and his proof that the $0-1$ polytope $P$ is found after $n$ steps relies on a nontrivial result of Curto and Fialkow (2000) about truncated moment sequences. The relaxations in Lasserre's hierarchy are defined in terms of moment matrices, which are matrices indexed by subsets of the set $V=\{1, \ldots, n\}$ and having the property that their $(I, J)$-entry depends only on the union $I \cup J$. In fact, the Sherali-Adams relaxations can also be formulated within this framework of moment matrices. The fact of formulating both Lasserre and Sherali-Adams constructions in a common setting permits a better understanding of how they relate; both constructions apply in fact to the case when $K$ is a semi-algebraic set contained in the cube $[0,1]^{n}$. Moreover, the same argument can be used for showing that the $0-1$ polytope $P$ is found after $n$ steps in both constructions. This argument concerns an elementary property of the zeta matrix of the lattice $\mathcal{P}(V)$, presented in Section 3.1. We show in Section 4 that the Lasserre hierarchy is a common refinement of the Sherali-Adams and Lovász-Schrijver hierarchies. We give in Section 5 two examples showing that $n$ steps are sometimes needed for finding $P$ when using the Sherali-Adams construction and we illustrate in Section 6 how the various methods apply to the stable set polytope and to the cut polytope of a graph. Section 7 contains some background information about the moment 
problem and the representation of positive polynomials as sums of squares, useful for understanding Lasserre's approach. In particular, we show that our presentation of Lasserre's method in Section 3 (in terms of moment matrices indexed by the semigroup $\mathcal{P}(V)$ ) is equivalent to the original presentation of Lasserre (in terms of moment matrices indexed by the semigroup $\mathbb{Z}_{+}^{n}$ ).

\section{The Lovász-Schrijver hierarchy}

Let $K$ be a convex body contained in the cube $[0,1]^{n}$ and let

$$
P:=\operatorname{conv}\left(K \cap\{0,1\}^{n}\right)
$$

be the $0-1$ polytope to be described. For convenience, define

$$
\tilde{K}:=\left\{\lambda\left(\begin{array}{l}
1 \\
x
\end{array}\right) \mid x \in K, \lambda \geq 0\right\},
$$

the homogenization of $K ; \tilde{K}$ is a cone in $\mathbb{R}^{n+1}$ (the additional coordinate is indexed by 0 ) and $K=$ $\left\{x \in \mathbb{R}^{n} \mid\left(\begin{array}{l}1 \\ x\end{array}\right) \in \tilde{K}\right\}$. Let $M(K)$ denote the set of symmetric matrices $Y=\left(y_{i j}\right)_{i, j=0}^{n}$ satisfying

$$
\begin{gathered}
y_{j, j}=y_{0, j} \text { for } j=1, \ldots, n, \\
Y e_{j}, Y\left(e_{0}-e_{j}\right) \in \tilde{K} \text { for } j=1, \ldots, n
\end{gathered}
$$

and set

$$
N(K):=\left\{x \in \mathbb{R}^{n} \mid\left(\begin{array}{l}
1 \\
x
\end{array}\right)=Y e_{0} \text { for some } Y \in M(K)\right\},
$$

where $e_{0}, e_{1}, \ldots, e_{n}$ denote the standard unit vectors in $\mathbb{R}^{n+1}$. Then,

$$
P \subseteq N(K) \subseteq K
$$

The inclusion $P \subseteq N(K)$ follows from the fact that, for $x \in K \cap\{0,1\}^{n}$, the matrix $Y:=\left(\begin{array}{l}1 \\ x\end{array}\right)\left(\begin{array}{l}1 \\ x\end{array}\right)^{T}$ belongs to $M(K)$ and the inclusion $N(K) \subseteq K$ follows from property (3). Define iteratively $N^{1}(K):=$ $N(K)$ and, for $t \geq 2, N^{t}(K):=N\left(N^{t-1}(K)\right)$. Then,

$$
P \subseteq N^{n}(K) \subseteq \ldots \subseteq N^{t+1}(K) \subseteq N^{t}(K) \subseteq \ldots \subseteq N(K) \subseteq K
$$

Lovász and Schrijver (1991) show that $N^{n}(K)=P$. (Their proof assumes that $K$ is a polytope but remains valid for any convex body $K$.)

Stronger relaxations are obtained by adding positive semidefiniteness. Set

$$
M_{+}(K):=\{Y \in M(K) \mid Y \succeq 0\} \text { and } N_{+}(K)=\left\{x \in \mathbb{R}^{n} \mid\left(\begin{array}{l}
1 \\
x
\end{array}\right)=Y e_{0} \text { for some } Y \in M_{+}(K)\right\} .
$$

Then,

$$
P \subseteq N_{+}(K) \subseteq K .
$$


The inclusion $P \subseteq N_{+}(K)$ follows from the fact that, for $x \in K \cap\{0,1\}^{n}$, the matrix $Y:=\left(\begin{array}{l}1 \\ x\end{array}\right)\left(\begin{array}{l}1 \\ x\end{array}\right)^{T}$ is positive semidefinite and thus belongs to $M_{+}(K)$. Define iteratively $N_{+}^{1}(K):=N_{+}(K)$ and $N_{+}^{t}(K):=$ $N_{+}\left(N_{+}^{t-1}(K)\right)$ for $t \geq 2$. Then,

$$
P \subseteq N_{+}^{t}(K) \subseteq N^{t}(K) \text { for } t \geq 1
$$

Lovász and Schrijver (1991) introduce in fact two relaxations $N(K, Q) \supseteq N(K, K)$ of $K$ (where $\left.Q=[0,1]^{n}\right)$. The set $N(K)$ considered here coincides with $N(K, Q)$; similarly, $N_{+}(K)=N_{+}(K, Q)$.

\section{The Sherali-Adams and Lasserre hierarchies}

The Sherali-Adams and Lasserre constructions apply to semi-algebraic sets contained in the cube $[0,1]^{n}$. Let

$$
K:=\left\{x \in[0,1]^{n} \mid g_{\ell}(x) \geq 0 \text { for } \ell=1, \ldots, m\right\}
$$

where $g_{1}, \ldots, g_{m}$ are polynomials in $x_{1}, \ldots, x_{n}$ and let $P:=\operatorname{conv}\left(K \cap\{0,1\}^{n}\right)$ be the $0-1$ polytope to be described. As $x_{i}^{2}=x_{i}(i=1, \ldots, n)$ for any $x \in\{0,1\}^{n}$, we can assume that each variable occurs in every polynomial $g_{\ell}$ with a degree $\leq 1$ and thus $g_{\ell}(x)$ can be written as

$$
\sum_{I \subseteq V} g_{\ell}(I) \prod_{i \in I} x_{i}
$$

Then the same symbol $g_{\ell}$ is used for denoting the vector in $\mathbb{R}^{\mathcal{P}(V)}$ with components $g_{\ell}(I)(I \subseteq V)$. We first present the two constructions in the common setting of moment matrices. For this, we need some definitions.

Given $V:=\{1, \ldots, n\}, \mathcal{P}(V)$ denotes the collection of all subsets of $V$ and, for $1 \leq t \leq n, \mathcal{P}_{t}(V)$ denotes the collection of subsets of cardinality $\leq t$. The components of a vector $y \in \mathbb{R}^{\mathcal{P}(V)}$ are denoted as $y_{I}$ or $y(I)$; we also set $y_{0}=y_{\emptyset}, y_{i}=y_{\{i\}}$ and $y_{i j}=y_{\{i, j\}}$. Given $y \in \mathbb{R}^{\mathcal{P}(V)}$, an integer $1 \leq t \leq n$, and a subset $U \subseteq V$, define the matrices

$$
M_{t}(y):=(y(I \cup J))_{|I|,|J| \leq t}, M_{U}(y):=(y(I \cup J))_{I, J \subseteq U} .
$$

Thus, $M_{V}(y)=M_{n}(y)$. The matrix $M_{V}(y)$ is known as the moment matrix of $y$. Moment matrices come up in the classic moment theory as well as the following product, often called shift operator in the literature (cf., e.g., Fuglede (1983)). For $x, y \in \mathbb{R}^{\mathcal{P}(V)}$, define the vector $x * y \in \mathbb{R}^{\mathcal{P}(V)}$ by

$$
x * y:=M_{V}(y) x ; \text { that is, } x * y(I)=\sum_{K \subseteq V} x_{K} y_{I \cup K} \text { for } I \subseteq V .
$$

One can easily verify the following commutation rule which will be used later in Section 4:

$$
x *(y * z)=y *(x * z) \text { for } x, y, z \in \mathbb{R}^{\mathcal{P}(V)} .
$$

More detailed information about moment theory will be given in Section 7.2. Examples of moment matrices arise from $0-1$ vectors in the following way. If $x \in\{0,1\}^{V}$ and $y:=\left(\prod_{i \in I} x_{i}\right)_{I \subseteq V}$, then 
$y y^{T}=M_{V}(y)$ is therefore a moment matrix. More generally, we have the following observation, which underlies the Sherali-Adams and Lasserre constructions.

Lemma 1. Given $x \in K \cap\{0,1\}^{n}$, the vector $y \in \mathbb{R}^{\mathcal{P}(V)}$ with entries $y(I):=\prod_{i \in I} x_{i}(I \subseteq V)$ satisfies

$$
M_{V}(y) \succeq 0, M_{V}\left(g_{\ell} * y\right) \succeq 0 \text { for } \ell=1, \ldots, m .
$$

Proof. Indeed, $M_{V}(y)=y y^{T}$ and $M_{V}\left(g_{\ell} * y\right)=g_{\ell}(x) y y^{T}$, since $y(I \cup J)=y(I) \cdot y(J)$ for all $I, J \subseteq V$.

One can relax the condition (8) and require positive semidefiniteness of certain principal submatrices of the moment matrices $M_{V}(y)$ and $M_{V}\left(g_{\ell} * y\right)$. Namely, Lasserre requires that

$$
M_{t+1}(y) \succeq 0, M_{t-v_{\ell}+1}\left(g_{\ell} * y\right) \succeq 0 \text { for } \ell=1, \ldots, m
$$

(for an integer $t \geq v_{\ell}-1$, where $v_{\ell}:=\left\lceil\frac{w_{\ell}}{2}\right\rceil, w_{\ell}$ being the degree of $g_{\ell}$ ) while Sherali and Adams require that

$$
\begin{gathered}
M_{W}(y) \\
\succeq 0 \text { for } W \subseteq V \text { with }|W|=\min (t+w, n) \\
M_{U}\left(g_{\ell} * y\right) \succeq 0 \text { for } U \subseteq V \text { with }|U|=t \text { and } \ell=1, \ldots, m
\end{gathered}
$$

(for an integer $t=1, \ldots, n$, where $w:=\max _{\ell} w_{\ell}$ ). The corresponding relaxations of $P$ are obtained by projecting the variable $y$ onto the subspace $\mathbb{R}^{n}$ indexed by the singletons in $\mathcal{P}(V)$. Sherali and Adams and Lasserre show that $P$ is found after $n$ steps in the two constructions. These two results are a direct consequence of Corollary 3 below (together with (12)) asserting that the cone in $\mathbb{R}^{\mathcal{P}(V)}$ consisting of the vectors $y$ satisfying (8) is generated by $0-1$ vectors.

The Sherali-Adams relaxations turn out to be linear relaxations since the condition (10) can be reformulated as a linear system in $y$ (cf. Lemma 2 below). We present in Section 3.2 the original definition of the Sherali-Adams relaxations given in (Sherali and Adams 1990) and its equivalence with the above definition.

\subsection{Preliminary results}

Let $Z$ denote the square $0-1$ matrix indexed by $\mathcal{P}(V)$ with entry $Z_{I, J}=1$ if and only if $I \subseteq J$. Its inverse $Z^{-1}$ has entries

$$
Z_{I, J}^{-1}=(-1)^{|J \backslash I|} \text { if } I \subseteq J, Z_{I, J}^{-1}=0 \text { otherwise. }
$$

The matrix $Z$ is known as the zeta matrix of the lattice $\mathcal{P}(V)$ and its inverse $Z^{-1}$ as the Möbius matrix of $\mathcal{P}(V)$ (cf. Wilf 1968). Let $\zeta^{J}$ denote the $J$-th column of $Z$; it has entries $\zeta^{J}(I)=\prod_{i \in I} x_{i}(I \subseteq V)$, setting $x:=\chi^{J}$. Given a subset $\mathcal{J} \subseteq \mathcal{P}(V)$, let

$$
\mathcal{C}_{\mathcal{J}}:=\left\{\sum_{J \in \mathcal{J}} \lambda_{J} \zeta^{J} \mid \lambda_{J} \geq 0 \text { for } J \in \mathcal{J}\right\}
$$


denote the cone generated by the columns of $Z$ indexed by $\mathcal{J}$. Hence, $\mathcal{C}_{\mathcal{J}}$ is a simplicial cone in $\mathbb{R}^{\mathcal{P}(V)}$ and

$$
\mathcal{C}_{\mathcal{J}}=\left\{y \in \mathbb{R}^{\mathcal{P}(V)} \mid Z^{-1} y \geq 0,\left(Z^{-1} y\right)_{J}=0 \forall J \notin \mathcal{J}\right\} .
$$

Lemma 2 and Corollary 3 below show how to reformulate membership in the cone $\mathcal{C}_{\mathcal{J}}$ in terms of positive semidefiniteness of certain moment matrices. This will be the key tool for proving the convergence in $n$ steps of the Sherali-Adams and Lasserre hierarchies. Lemma 2 is based on ideas from Section 3.a in (Lovász and Schrijver 1991).

Lemma 2. Let $g, y \in \mathbb{R}^{\mathcal{P}(V)}$. Then,

(i) $M_{V}(g * y) \succeq 0 \Longleftrightarrow\left(Z^{-1} y\right)_{H} \cdot g^{T} \zeta^{H} \geq 0$ for all $H \subseteq V$.

(ii) $M_{V}(y) \succeq 0 \Longleftrightarrow Z^{-1} y \geq 0 \Longleftrightarrow \sum_{H \supseteq I}(-1)^{|H \backslash I|} y(H) \geq 0$ for all $I \subseteq V$.

Proof. (i) Let $u \in \mathbb{R}^{\mathcal{P}(V)}$ with entries $u_{H}:=\left(Z^{-1} y\right)_{H} \cdot g^{T} \zeta^{H}(H \subseteq V)$, and let $D_{u}$ denote the diagonal matrix indexed by $\mathcal{P}(V)$ with diagonal entries $u_{H}(H \subseteq V)$. We show that $Z D_{u} Z^{T}=M_{V}(g * y)$. For this note that, for $H \subseteq V$,

$$
u_{H}=\left(Z^{-1} y\right)_{H} \cdot g^{T} \zeta^{H}=\left(\sum_{R \supseteq H}(-1)^{|R \backslash H|} y_{R}\right) \cdot\left(\sum_{K \subseteq H} g_{K}\right)=\sum_{K \subseteq H \subseteq R}(-1)^{|R \backslash H|} y_{R} g_{K} .
$$

Therefore, given $I, J \subseteq V$, the $(I, J)$-th entry of $Z D_{u} Z^{T}$ is equal to

$$
\sum_{H} Z_{I H} Z_{J H} u_{H}=\sum_{H \supseteq I \cup J} u_{H}=\sum_{K, R} y_{R} g_{K}\left(\sum_{I \cup J \cup K \subseteq H \subseteq R}(-1)^{|R \backslash H|}\right)=\sum_{K} g_{K} y_{I \cup J \cup K}=g * y(I \cup J)
$$

and thus to $M_{V}(g * y)_{I J}$, using the fact that $\sum_{I \cup J \cup K \subseteq H \subseteq R}(-1)^{|R \backslash H|}=1$ if $R=I \cup J \cup K$ and 0 otherwise. Assertion (i) now follows from the fact that $u \geq 0$ is equivalent to $D_{u} \succeq 0$.

The first equivalence in (ii) follows directly from (i) applied to $g$ with all zero components except $g_{\emptyset}=1$ and the second equivalence follows from the description of $Z^{-1}$ in (11).

Let $g_{\ell}(x)(\ell=1, \ldots, m)$ be polynomials in which every variable occurs with degree $\leq 1$ and set

$$
\mathcal{J}:=\left\{J \subseteq V \mid g_{\ell}^{T} \zeta^{J} \geq 0 \text { for all } \ell=1, \ldots, m\right\}=\left\{J \subseteq V \mid g_{\ell}\left(\chi^{J}\right) \geq 0 \text { for all } \ell=1, \ldots, m\right\} .
$$

In the case $\mathcal{J}=\mathcal{P}(V)$, the next result is given in (Lovász and Schrijver 1991) and (Sherali and Adams 1990).

Corollary 3. $\mathcal{C}_{\mathcal{J}}=\left\{y \in \mathbb{R}^{\mathcal{P}(V)} \mid M_{V}(y) \succeq 0\right.$ and $M_{V}\left(g_{\ell} * y\right) \succeq 0$ for all $\left.\ell=1, \ldots, m\right\}$.

Proof. Let $y \in \mathbb{R}^{\mathcal{P}(V)}$. By (13), $y \in \mathcal{C}_{\mathcal{J}}$ if and only if $Z^{-1} y \geq 0$ and $\left(Z^{-1} y\right)_{J}=0$ for $J \notin \mathcal{J}$. Using (14), this is equivalent to $Z^{-1} y \geq 0$ and $\left(Z^{-1} y\right)_{J} \cdot g_{\ell}^{T} \zeta^{J} \geq 0$ for all $\ell=1, \ldots, m$ and $J \subseteq V$. Therefore, 
using Lemma 2, $y \in \mathcal{C}_{\mathcal{J}}$ if and only if $M_{V}(y) \succeq 0$ and $M_{V}\left(g_{\ell} * y\right) \succeq 0$ for $\ell=1, \ldots, m$.

Example 4. We illustrate Lemma 2 and Corollary 3 on a small example. For $V=\{1,2\}$, consider the cone $\mathcal{C}_{\mathcal{J}}$ where $\mathcal{J}$ is the set of $0-1$ solutions of the inequality $g(x):=x_{1}+x_{2}-1 \geq 0$. Then,

$$
\begin{gathered}
Z=\left(\begin{array}{llll}
1 & 1 & 1 & 1 \\
0 & 1 & 0 & 1 \\
0 & 0 & 1 & 1 \\
0 & 0 & 0 & 1
\end{array}\right), Z^{-1}=\left(\begin{array}{cccc}
1 & -1 & -1 & 1 \\
0 & 1 & 0 & -1 \\
0 & 0 & 1 & -1 \\
0 & 0 & 0 & 1
\end{array}\right), \\
M_{V}(y)=\left(\begin{array}{cccc}
y_{0} & y_{1} & y_{2} & y_{12} \\
y_{1} & y_{1} & y_{12} & y_{12} \\
y_{2} & y_{12} & y_{2} & y_{12} \\
y_{12} & y_{12} & y_{12} & y_{12}
\end{array}\right), M_{V}(g * y)=\left(\begin{array}{cccc}
-y_{0}+y_{1}+y_{2} & y_{12} & y_{12} & y_{12} \\
y_{12} & y_{12} & y_{12} & y_{12} \\
y_{12} & y_{12} & y_{12} & y_{12} \\
y_{12} & y_{12} & y_{12} & y_{12}
\end{array}\right)
\end{gathered}
$$

since $g * y$ has all its entries equal to $y_{12}$ except its $\emptyset$-entry equal to $-y_{0}+y_{1}+y_{2}$. Therefore,

$$
\begin{gathered}
M_{V}(y) \succeq 0 \Longleftrightarrow y_{0}-y_{1}-y_{2}+y_{12} \geq 0, y_{1}-y_{12} \geq 0, y_{2}-y_{12} \geq 0, y_{12} \geq 0, \\
M_{V}(g * y) \succeq 0 \Longleftrightarrow-y_{0}+y_{1}+y_{2}-y_{12} \geq 0, y_{12} \geq 0 .
\end{gathered}
$$

Hence the cone $\mathcal{C}_{\mathcal{J}}$ is described by the linear system:

$$
\begin{aligned}
y_{0}-y_{1}-y_{2}+y_{12} & =0 \\
y_{1}-y_{12} & \geq 0 \\
y_{2}-y_{12} & \geq 0 \\
y_{12} & \geq 0
\end{aligned}
$$

and its projection on the subspace $\mathbb{R}^{2}$ indexed by variables $y_{1}$ and $y_{2}$ (obtained by eliminating variable $y_{12}$ and setting $y_{0}=1$ ) is the polytope defined by

$$
y_{1}+y_{2} \geq 1,1 \geq y_{1}, 1 \geq y_{2} .
$$

We conclude with a result showing how positive semidefiniteness of the moment matrices of $g * y$, when $g(x)$ is one of the polynomials $x_{i}, 1-x_{i}(i=1, \ldots, n)$, can be reformulated in terms of positive semidefiniteness of the moment matrix of $y$. This result tells us how to handle the bound inequalities $0 \leq x_{i} \leq 1$ and will be used in Section 4 for the proof of Theorem 14 .

Lemma 5. Let $y \in \mathbb{R}^{\mathcal{P}(V)}$ and $1 \leq t \leq n$ an integer. If $M_{t}(y) \succeq 0$ then, for all $i=1, \ldots, n$, $M_{t-1}\left(e_{i} * y\right), M_{t-1}\left(\left(e_{\emptyset}-e_{i}\right) * y\right) \succeq 0$.

Proof. Set $\mathcal{P}_{1}:=\{I \subseteq V|| I \mid \leq t-2, i \notin I\}, \mathcal{P}_{2}:=\{I \subseteq V|| I \mid=t-1, i \notin I\}$, and $\mathcal{P}_{j}^{\prime}:=\left\{I \cup\{i\} \mid I \in \mathcal{P}_{j}\right\}$ for $j=1,2$ and let $Y$ denote the principal submatrix of $M_{t}(y)$ indexed by 


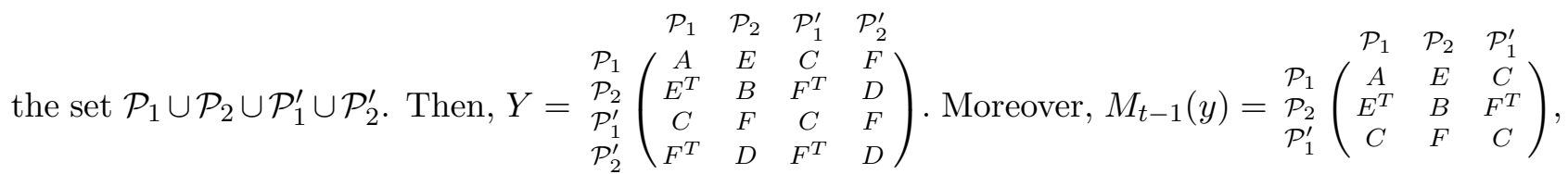

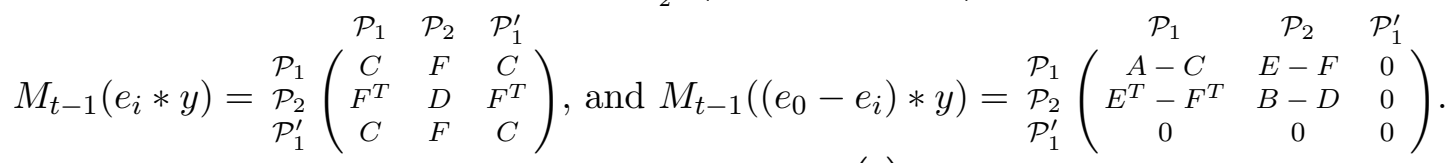

Therefore, we have that $\left(x^{T} y^{T} z^{T}\right) M_{t-1}\left(e_{i} * y\right)\left(\begin{array}{l}x \\ y \\ z\end{array}\right)=\left((x+z)^{T} y^{T}\right)\left(\begin{array}{cc}C & F \\ F^{T} & D\end{array}\right)\left(\begin{array}{c}x+z \\ y\end{array}\right) \geq 0$ and $\left(\begin{array}{lll}x^{T} & y^{T} z^{T}\end{array}\right) M_{t-1}\left(\left(e_{\emptyset}-e_{i}\right) * y\right)\left(\begin{array}{l}x \\ y \\ z\end{array}\right)=\left(\begin{array}{lll}x^{T} & y^{T}-x^{T}-y^{T}\end{array}\right) Y\left(\begin{array}{c}x \\ y \\ -x \\ -y\end{array}\right) \geq 0$ for all $x, y, z$, since the matrices $\left(\begin{array}{cc}C & F \\ F^{T} & D\end{array}\right)$ and $Y$ are positive semidefinite. This shows that $M_{t-1}\left(e_{i} * y\right), M_{t-1}\left(\left(e_{\emptyset}-e_{i}\right) * y\right) \succeq 0$.

\subsection{The Sherali-Adams hierarchy}

Let $K$ be a semi-algebraic set as in (4), where the $g_{\ell}$ 's are polynomials in which every variable occurs with degree at most 1 and let $P=\operatorname{conv}\left(K \cap\{0,1\}^{n}\right)$ be the polytope to be described. Let $\mathcal{J}$ be as in relation (14). Let $w_{\ell}$ denote the degree of the polynomial $g_{\ell}$ and set

$$
v_{\ell}:=\left\lceil\frac{w_{\ell}}{2}\right\rceil, w:=\max w_{\ell}, v:=\max v_{\ell} .
$$

When there is no constraint $g_{\ell}(x) \geq 0$ in the definition of $K, K$ is the cube $[0,1]^{n}$ and we set $v=w:=0$. We now introduce the Sherali-Adams relaxations as linear relaxations and then observe that they can be reformulated as the semidefinite programs (10). Let $t \in\{1, \ldots, n\}$. Multiply each inequality $g_{\ell}(x) \geq 0(\ell=1, \ldots, m)$ by each product

$$
f(I, J):=\prod_{i \in I} x_{i} \cdot \prod_{j \in J}\left(1-x_{j}\right)
$$

where $I, J$ are disjoint subsets of $V=\{1, \ldots, n\}$ such that $|I \cup J|=t$. In this way, we obtain a set of inequalities that are still valid for $P$. Add to this set all the inequalities $f(I, J) \geq 0$ where $I, J$ are disjoint subsets with $|I \cup J|=\min (t+w, n)$. Replace each square $x_{i}^{2}$ by $x_{i}$ and linearize the product $\prod_{i \in I} x_{i}$ by a new variable $y_{I}$ for $I \subseteq V$ (thus setting $y_{i}=x_{i}$ for $i \in V$ ). This defines a set $R_{t}(K)$ in the space $\mathbb{R}^{\mathcal{P}_{t+w}(V)}$. As

$$
\prod_{i \in I} x_{i} \cdot \prod_{j \in J}\left(1-x_{j}\right)=\sum_{I \subseteq H \subseteq I \cup J}(-1)^{|H \backslash I|} \prod_{h \in H} x_{h}
$$

the quantity obtained by linearizing $g_{\ell}(x) \prod_{i \in I} x_{i} \cdot \prod_{j \in J}\left(1-x_{j}\right)$ reads

$$
\left(\sum_{K \subseteq V} g_{\ell}(K) \prod_{k \in K} x_{k}\right) \cdot\left(\sum_{I \subseteq H \subseteq I \cup J}(-1)^{|H \backslash I|} \prod_{h \in H} x_{h}\right)=\sum_{I \subseteq H \subseteq I \cup J}(-1)^{|H \backslash I|} g_{\ell} * y(H) .
$$


Therefore, $R_{t}(K)$ consists of the vectors $y \in \mathbb{R}^{\mathcal{P}_{t+w}(V)}$ satisfying the inequalities:

$$
\begin{gathered}
\sum_{I \subseteq H \subseteq U}(-1)^{|H \backslash I|} g_{\ell} * y(H) \geq 0 \text { for all } \ell=1, \ldots, m \text { and all } I \subseteq U \subseteq V \text { with }|U|=t, \\
\sum_{I \subseteq H \subseteq W}(-1)^{|H \backslash I|} y(H) \geq 0 \text { for all } I \subseteq W \subseteq V \text { with }|W|=\min (t+w, n) .
\end{gathered}
$$

Using Lemma 2, the set $R_{t}(K)$ can be reformulated as

$$
\begin{aligned}
R_{t}(K)=\left\{y \in \mathbb{R}^{\mathcal{P}_{t+w}(V)} \mid\right. & M_{U}\left(g_{\ell} * y\right) \succeq 0 \text { for all } U \subseteq V \text { with }|U|=t \text { and } \ell=1, \ldots, m \\
& \left.M_{W}(y) \succeq 0 \text { for all } W \subseteq V \text { with }|W|=\min (t+w, n)\right\} .
\end{aligned}
$$

In view of Corollary 3 we find that

$$
R_{n}(K)=\mathcal{C}_{\mathcal{J}}
$$

Let $S_{t}(K)$ denote the projection of $R_{t}(K) \cap\left\{y \mid y_{\emptyset}=1\right\}$ on the subspace $\mathbb{R}^{n}$ indexed by the singletons. By the above, we deduce that

$$
P=S_{n}(K) \subseteq \ldots \subseteq S_{t+1}(K) \subseteq S_{t}(K) \subseteq \ldots \subseteq S_{1}(K) .
$$

In general, the set $S_{1}(K)$ is not contained in $K$; this is due to the fact that $S_{1}(K)$ is convex while $K$ need not be convex. (As a nonconvex example, consider $K=\left\{x \in[0,1]^{2} \mid x_{1}+x_{2}-x_{1} x_{2} \geq 1\right\}$ which is the union of two intervals, $K=\left\{x \in[0,1]^{2} \mid x_{1}=1\right.$ or $\left.x_{2}=1\right\}$, while $P=\left\{x \in[0,1]^{2} \mid x_{1}+x_{2} \geq 1\right\}$.) In the linear case, i.e., when all polynomials $g_{\ell}$ have degree 1 , then $K$ is convex and $S_{1}(K) \subseteq K$.

Remark 6. Note that the inequalities (17) (resp., (18)) remain valid for $R_{t}(K)$ for any $U$ with $|U| \leq t$ (resp., any $W$ with $|W| \leq \min (t+w, n)$ ). This follows from the fact that equality $f(I, J)=$ $f(I \cup\{k\}, J)+f(I, J \cup\{k\})$ holds for any element $k \in V \backslash I \cup J$ and any disjoint $I, J \subseteq V$.

Another observation is that the conditions: $f(I, J) \geq 0$ for all disjoint $I, J \subseteq V$ with $|I \cup J|=$ $\min (t+w, n)$ yield (after linearization and replacing squares $x_{i}^{2}$ by $x_{i}$ ) the same solution set as the conditions: $f(I, J) \cdot f(R, S) \geq 0$ for all disjoint $I, J \subseteq V$ with $|I \cup J|=t$ and all disjoint $R, S \subseteq V$ with $|R \cup S|=w$. Hence we have the following result (compare with Lemma 5), to be used later in the proof of Theorem 12.

Lemma 7. Given $y \in \mathbb{R}^{\mathcal{P}(V)}$ and an integer $1 \leq t \leq n$, the following assertions are equivalent.

(i) $M_{U}\left(e_{i} * y\right), M_{U}\left(\left(e_{\emptyset}-e_{i}\right) * y\right) \succeq 0$ for all $U \subseteq V$ with $|U|=t$ and all $i=1, \ldots, n$

(ii) $M_{W}(y) \succeq 0$ for all $W \subseteq V$ with $|W|=\min (n, t+1)$.

Remark 8. Matrix reformulation. Let $\mathcal{K}$ denote the linearization of $K$ consisting of the vectors $y \in \mathbb{R}^{\mathcal{P}_{w}(V)}$ satisfying the linear system:

$$
\begin{aligned}
& g_{\ell}^{T} y \geq 0 \text { for } \ell=1, \ldots, m, \\
& \sum_{R \subseteq H \subseteq S}(-1)^{|H \backslash R|} y_{H} \geq 0 \text { for all } R \subseteq S \subseteq V \text { with }|S|=w .
\end{aligned}
$$


Given $y \in \mathbb{R}^{\mathcal{P}_{t+w}(V)}$, consider the matrix $Y$ whose rows and columns are indexed, respectively, by $\mathcal{P}_{w}(V)$ and $\mathcal{P}_{t}(V)$ and with entries $Y(K, H):=y(K \cup H)$ for $K \in \mathcal{P}_{w}(V)$ and $H \in \mathcal{P}_{t}(V)$. Denote by $e_{H}\left(H \in \mathcal{P}_{t}(V)\right)$ the elementary unit vectors in $\mathbb{R}^{\mathcal{P}_{t}(V)}$; then $Y e_{H}$ is the column of $Y$ indexed by $H$. Then,

$$
y \in R_{t}(K) \Longleftrightarrow Y\left(\sum_{I \subseteq H \subseteq U}(-1)^{|H \backslash I|} e_{H}\right) \in \mathcal{K} \text { for all } I \subseteq U \subseteq V \text { with }|U|=t .
$$

In particular, in the case $t=1, y \in R_{1}(K) \Longleftrightarrow Y e_{j}, Y\left(e_{\emptyset}-e_{j}\right) \in \mathcal{K}$ for all $j \in V$. Note the analogy with the relation (3) defining the matrix set $M(K)$ in the Lovász-Schrijver procedure.

In the linear case $w=1$, the second set of inequalities in (20) reads: $0 \leq y \leq y_{0}(i \in V)$ and thus the cone $\mathcal{K}$ coincides with the set $\tilde{K}$ introduced in (1). Therefore, we find the well known fact that $S_{1}(K)=N(K)$; that is, the first steps in the Sherali-Adams and Lovász-Schrijver procedures coincide. The next steps are however distinct (although there is an inclusion relationship; see Corollary 13). A main difference between the two methods is that the Lovász-Schrijver procedure constructs the successive relaxations recursively by applying $t$ successive lift-and-project steps, each taking place in a space of dimension $O\left(n^{2}\right)$, whereas the Sherali-Adams procedure carries out only one direct lifting step, occurring in a space of dimension $O\left(n^{t+1}\right)$. Moreover, the projection step is not mandatory in the Sherali-Adams procedure, as approximate solutions for optimization problems on $P$ can be obtained by optimizing directly over the set $R_{t}(K)$.

Remark 9. The original construction of Sherali and Adams (known as the reformulation-linearization technique or RLT) applies more generally to 0-1 mixed integer polynomial programs which are linear in the continuous variables (Sherali and Adams 1994). However, it is not clear whether the presen-

tation given here in terms of moment matrices extends to the mixed integer case. Strengthenings of the basic RLT method have been proposed for general polynomial programming problems involving, in particular, taking products of other inequalities than the bound-factors $x_{i} \geq 0$ and $1-x_{i} \geq 0$ (see Sherali and Tuncbilek (1992,1997), Sherali, Adams and Driscoll (1998), Sherali and Adams (1999)). We will come back to it in Section 7.3.

\subsection{The Lasserre hierarchy}

For $t \geq v-1$, with $v$ defined as in (15), set

$$
P_{t}(K):=\left\{y \in \mathbb{R}^{\mathcal{P}_{2 t+2}(V)} \mid M_{t+1}(y) \succeq 0, M_{t+1-v_{\ell}}\left(g_{\ell} * y\right) \succeq 0 \text { for } \ell=1, \ldots, m\right\}
$$

and define $Q_{t}(K)$ as the projection of $P_{t}(K) \cap\left\{y \mid y_{\emptyset}=1\right\}$ on the subspace $\mathbb{R}^{n}$ indexed by the singletons. Therefore,

$$
P \subseteq Q_{n+v-1}(K) \subseteq \ldots \subseteq Q_{v}(K) \subseteq Q_{v-1}(K)
$$

Lasserre (2001b) shows that

$$
P=Q_{n+v-1}(K) .
$$

This result follows, in fact, as a direct consequence of Corollary 3 , since $P_{n+v-1}(K)=\mathcal{C}_{\mathcal{J}}$. The following inclusion relationship holds between the Lasserre hierarchy and the Sherali-Adams hierarchy. 
Proposition 10. For any $t=1, \ldots, n, Q_{t+w-1}(K) \subseteq S_{t}(K)$.

Proof. Let $y \in P_{t+w-1}(K)$; that is, $y \in \mathbb{R}^{\mathcal{P}_{2 t+2 w}(V)}$ satisfies $M_{t+w}(y) \succeq 0$ and $M_{t+w-v_{\ell}}\left(g_{\ell} * y\right) \succeq 0$ for $\ell=1, \ldots, m$. We verify that the restriction of $y$ to $\mathbb{R}^{\mathcal{P}_{t+w}(V)}$ belongs to $R_{t}(K)$. Indeed, given $U, W \subseteq V$ with $|U|=t$ and $|W|=\min (t+w, n), M_{W}(y) \succeq 0$ since it is a principal submatrix of $M_{t+w}(y)$ and $M_{U}\left(g_{\ell} * y\right) \succeq 0$ since it is a principal submatrix of $M_{t+w-v_{\ell}}\left(g_{\ell} * y\right)$.

Remark 11. One could weaken the definition of the set $R_{t}(K)$ by replacing the condition $|W|=$ $\min (t+w, n)$ by $|W|=\min (t+1, n)$ (in (18) and (19)); denote by $R_{t}^{\prime}(K)$ the set obtained in this way and by $S_{t}^{\prime}(K)$ the projection on $\mathbb{R}^{n}$ of $R_{t}^{\prime}(K) \cap\left\{y \mid y_{\emptyset}=1\right\}$. Thus $R_{t}(K) \subseteq R_{t}^{\prime}(K)$ and $S_{t}(K) \subseteq S_{t}^{\prime}(K)$. Equality $R_{n}^{\prime}(K)=\mathcal{C}_{\mathcal{J}}$ still holds, yielding $S_{n}^{\prime}(K)=P$. Hence the weaker hierarchy $S_{t}^{\prime}(K)$ also converges to $P$ in $n$ steps. In the linear case $w=1$, the two definitions coincide: $S_{t}(K)=S_{t}^{\prime}(K)$. One can verify the inclusion

$$
Q_{t+v-1}(K) \subseteq S_{t}^{\prime}(K)
$$

(for $w \geq 1$ ) which implies again the convergence result: $P=Q_{n+v-1}(K)$ for the Lasserre hierarchy.

The construction of Lasserre is originally presented in terms of moment matrices indexed by integer sequences (rather than subsets of $V$ ) and the proof of convergence uses results about moment sequences and the representation of positive polynomials as sums of squares. We review Lasserre's approach in Section 7 and show that it is equivalent to the above presentation.

\section{Comparing the Lasserre, Sherali-Adams and Lovász-Schrijver Re- laxations}

We assume in this section that $K$ is a polytope; that is, $K$ is defined by (4) where all the polynomials $g_{\ell}$ have degree 1 (thus $v=w=1$, or $v=w=0$ if $K=[0,1]^{n}$ ). As recalled earlier (in Remark 8), the first steps of the Sherali-Adams and Lovász-Schrijver hierarchies are then identical; that is, $S_{1}(K)=N(K)$. It follows from results in (Lovász and Schrijver 1991) that $S_{t}(K) \subseteq N^{t}(K)$; that is, the Sherali-Adams hierarchy refines the Lovász-Schrijver hierarchy. This inclusion also follows from Theorem 12 below which shows a stronger result and has a simple direct proof (while the proof in (Lovász and Schrijver 1991) is more involved).

Theorem 12. If $K$ is a polytope, then $S_{t}(K) \subseteq N\left(S_{t-1}(K)\right)$ for all $t=1, \ldots, n$ (setting $\left.S_{0}(K):=K\right)$.

Proof. Let $t \geq 2$ and let $\left(y_{1}, \ldots, y_{n}\right)^{T} \in S_{t}(K)$; that is, $\left(y_{1}, \ldots, y_{n}\right)^{T}$ is the projection of some $y \in R_{t}(K)$ with $y_{\emptyset}=1$. We show that the matrix $Y:=M_{1}(y)=\left(y_{I \cup J}\right)_{|I|,|J| \leq 1}$ belongs to $M\left(S_{t-1}(K)\right)$; that is, $Y e_{k}, Y\left(e_{\emptyset}-e_{k}\right)$ belong to $\widetilde{S_{t-1}(K)}$, the homogenization of $S_{t-1}(K)$, for all $k=1, \ldots, n$. As $Y e_{k}$ (resp., $\left.Y\left(e_{\emptyset}-e_{k}\right)\right)$ is the projection on $\mathbb{R}^{\mathcal{P}_{1}(V)}$ of the vector $e_{k} * y$ (resp., $\left.\left(e_{\emptyset}-e_{k}\right) * y\right)$, it suffices to show that $e_{k} * y$ and $\left(e_{\emptyset}-e_{k}\right) * y$ belong to $R_{t-1}(K)$. In other words, we have to show that $M_{W}\left(e_{k} * y\right)$, 
$M_{W}\left(\left(e_{\emptyset}-e_{k}\right) * y\right), M_{U}\left(g_{\ell} *\left(e_{k} * y\right)\right), M_{U}\left(g_{\ell} *\left[\left(e_{\emptyset}-e_{k}\right) * y\right]\right) \succeq 0$ for all $\ell=1, \ldots, m, U, W \subseteq V$ with $|U|=t-1,|W|=t$. This follows directly from the assumption that $y \in R_{t}(y)$ together with Lemma 7 and the commutation rule (7). (We have assumed here that $w=1$ but the proof is analogous for $w=0$.

Corollary 13. $S_{t}(K) \subseteq N^{t}(K)$ for all $t=1, \ldots, n$.

Proof. Directly from Theorem 12 using induction on $t$.

Proposition 10 and Corollary 13 imply that

$$
Q_{t}(K) \subseteq S_{t}(K) \subseteq N^{t}(K)
$$

for $t=1, \ldots, n$. In fact, one can show that the Lasserre hierarchy also refines the Lovász-Schrijver hierarchy obtained using the $N_{+}$operator.

Observe that $M(K)$ can be alternatively viewed as the set of matrices $Y:=M_{1}(y)$ where $y \in \mathbb{R}^{\mathcal{P}_{2}(V)}$ for which $Y e_{k}, Y\left(e_{0}-e_{k}\right) \in \tilde{K}$, i.e., $g_{\ell}^{T} Y e_{k}, g_{\ell}^{T} Y\left(e_{\emptyset}-e_{k}\right) \geq 0$ for all $\ell=1, \ldots, m$ and $k=1, \ldots, n$. As $g_{\ell}^{T} Y e_{0}=g_{\ell} * y(\emptyset), g_{\ell}^{T} Y e_{k}=g_{\ell} * y(k)$, the latter holds if and only if the principal submatrix of $M_{1}\left(g_{\ell} * y\right)$ indexed by $\emptyset$ and $\{k\}$ is positive semidefinite. In comparison, membership in $Q_{0}(K)$ requires only that $g_{\ell} * y(\emptyset) \geq 0$ for all $\ell$, while membership in $Q_{1}(K)$ requires that $M_{1}\left(g_{\ell} * y\right) \succeq 0$ for all $\ell$. Therefore, we have the following inclusions:

$$
Q_{1}(K) \subseteq N_{+}(K) \subseteq Q_{0}(K)
$$

Theorem 14. If $K$ is a polytope, then $Q_{t}(K) \subseteq N_{+}\left(Q_{t-1}(K)\right)$ for all $t=1, \ldots, n$.

Proof. Let $\left(y_{1}, \ldots, y_{n}\right)^{T} \in Q_{t}(K)$; that is, $\left(y_{1}, \ldots, y_{n}\right)^{T}$ is the projection of some $y \in P_{t}(K)$ with $y_{\emptyset}=1$. Set $Y:=M_{1}(y)$. We show that $Y e_{k}, Y\left(e_{\emptyset}-e_{k}\right) \in \widetilde{Q_{t-1}(K)}$, the homogenization of $Q_{t-1}(K)$, for $k=1, \ldots, n$. As $Y e_{k}$ (resp., $Y\left(e_{\emptyset}-e_{k}\right)$ ) is the projection on $\mathbb{R}^{\mathcal{P}_{1}(V)}$ of $e_{k} * y$ (resp., $\left.\left(e_{\emptyset}-e_{k}\right) * y\right)$, it suffices to show that $e_{k} * y$ and $\left(e_{\emptyset}-e_{k}\right) * y$ belong to $P_{t-1}(K)$. In other words, we have to show that $M_{t}\left(e_{k} * y\right), M_{t}\left(\left(e_{\emptyset}-e_{k}\right) * y\right), M_{t-1}\left(g_{\ell} *\left(e_{k} * y\right)\right), M_{t-1}\left(g_{\ell} *\left[\left(e_{\emptyset}-e_{k}\right) * y\right]\right) \succeq 0$ for all $\ell=1, \ldots, m$. This follows directly from the assumption that $y \in P_{t}(K)$ together with Lemma 5 and the commutation rule $(7)$.

Corollary 15. If $K$ is a polytope, then $Q_{t}(K) \subseteq N_{+}^{t}(K)$ for all $t=1, \ldots, n$.

Proof. Directly from Theorem 14 and (23) using induction on $t$.

An algorithmic comparison. Summarizing, we have:

$$
Q_{t}(K) \subseteq S_{t}(K) \cap N_{+}^{t}(K)
$$


for any $t=1, \ldots, n$. Therefore, the Lasserre set $Q_{t}(K)$ provides the sharpest relaxation of $P$. From an algorithmic point of view, it is however less well behaved than the Sherali-Adams and Lovász-Schrijver relaxations.

Given a convex body $B \subseteq \mathbb{R}^{n}$, the separation problem for $B$ is the problem of determining whether a given vector $y \in \mathbb{R}^{n}$ belongs to $B$ and, if not, of finding a hyperplane separating $y$ from $B$; the weak separation problem is the analogue problem where one allows for numerical errors. An important consequence of the ellipsoid method is that, if one can solve the weak separation problem for $B$ in polynomial time, then one can optimize any linear objective function over $B$ in polynomial time (with an arbitrary precision) and vice versa (assuming some technical information about $B$ like the knowledge of a ball contained in $B$ and of a ball containing $B$ ); see (Grötschel, Lovász and Schrijver 1988) for details.

If one can solve the weak separation problem for $K$ in polynomial time, then the same holds for $M(K)$ and $M_{+}(K)$ and thus for the projections $N(K)$ and $N_{+}(K)$. Therefore, one can optimize a linear objective function in polynomial time over the relaxations $N^{t}(K), N_{+}^{t}(K), S_{t}(K)$ for any fixed $t$; this is observed in (Lovász and Schrijver 1991) for the LS sets and the same argument works for the SA sets in view of the matrix reformulation (21) of the SA method. The assumption made over $K$ is trivially satisfied if $m$ is polynomial in $n$ but it may sometimes be satisfied even if $m$ is exponential in $n$. On the other hand, in order to claim that one can optimize over $Q_{t}(K)$ in polynomial time, one needs to assume that $m$ is polynomial in $n$, since the system defining $Q_{t}(K)$ involves $m$ LMI's associated to the inequalities of the linear system defining $K$.

Note that one can optimize over the Sherali-Adams and Lasserre sets without carrying out explicitely the projection step, whereas the Lovász-Schrijver relaxations intrinsically need a sequence of successive projections.

\section{The rank of the Sherali-Adams Procedure}

We present here two examples of a polytope $K \subseteq[0,1]^{n}$ for which $n$ iterations of the Sherali-Adams procedure are needed for finding the integer polytope $P=\operatorname{conv}\left(K \cap\{0,1\}^{n}\right)$.

Example 16. Let

$$
K:=\left\{x \in[0,1]^{n} \mid \sum_{r \in R}\left(1-x_{r}\right)+\sum_{r \in V \backslash R} x_{r} \geq \frac{1}{2} \text { for all } R \subseteq\{1, \ldots, n\}\right\} ;
$$

then $P=\emptyset$. We show in Proposition 17 below that $S_{n-1}(K) \neq \emptyset$, which implies that $P \neq S_{n-1}(K)$. The polytope $K$ has been used earlier to show that $n$ iterations are needed for the following procedures: taking Chvátal cuts (Chvátal, Cook and Hartman 1989), the $N_{+}$operator (Goemans and Tunçel 2001), the $N_{+}$operator combined with taking Chvátal cuts (Cook and Dash 2001), and the $N_{+}$operator combined with taking Gomory mixed integer cuts (equivalent to disjunctive cuts) (Cornuéjols and Li 2001). The following (easy to verify) identities will be used in the proof:

$$
\sum_{K \subseteq A} \frac{(-1)^{|K|}}{2^{|K|}}=\frac{1}{2^{|A|}}, \quad \sum_{K \subseteq A}|K| \frac{(-1)^{|K|}}{2^{|K|}}=-\frac{|A|}{2^{|A|}}
$$


for any set $A$. (For the second one, use the fact that $k\left(\begin{array}{l}n \\ k\end{array}\right)=n\left(\begin{array}{l}n-1 \\ k-1\end{array}\right)$.)

Proposition 17. Let $y \in \mathbb{R}^{\mathcal{P}(V)}$ with entries $y_{I}:=\frac{1}{2^{|I|}}(I \subseteq V)$. Then, $y \in R_{n-1}(K)$ where $K$ is defined by (24).

Proof. Let $v_{R} \in \mathbb{R}^{\mathcal{P}(V)}$ be the vector of coefficients of an inequality defining $K$, with all components zero except $v_{R}(\emptyset)=-\frac{1}{2}+|R|, v_{R}(r)=-1$ if $r \in R, v_{R}(r)=1$ if $r \in V \backslash R$, where $R$ is a given subset of $V$. Then, for $H \subseteq V$,

$$
\begin{aligned}
v_{R} * y(H) & =\left(|R|-\frac{1}{2}\right) y(H)-\sum_{r \in R} y(H \cup\{r\})+\sum_{r \in V \backslash R} y(H \cup\{r\}) \\
& =\frac{1}{2^{|H|}}\left(|R|-\frac{1}{2}+|H \backslash R|-|R \cap H|\right)+\frac{1}{2^{|H|+1}}(|V \backslash(H \cup R)|-|R \backslash H|) \\
& =\frac{1}{2^{|H|+1}}(n-1+|H|-2|R \cap H|) .
\end{aligned}
$$

Given a subset $U \subseteq V$ with $|U|=n-1$ and $I \subseteq U$, we have:

$$
\begin{aligned}
\varphi:= & \sum_{I \subseteq H \subseteq U}(-1)^{|H \backslash I|}\left(v_{R} * y\right)(H)=\frac{n-1}{2^{|I|+1}} \sum_{K \subseteq U \backslash I} \frac{(-1)^{|K|}}{2^{|K|}} \\
& +\frac{1}{2^{|I|+1}} \sum_{K \subseteq U \backslash I} \frac{(-1)^{|K|}}{2^{|K|}}(|I|+|K|)-\frac{1}{2^{|I|+1}} \sum_{K \subseteq U \backslash I} \frac{(-1)^{|K|}}{2^{|K|}}(|R \cap I|+|R \cap K|) .
\end{aligned}
$$

Using (25), one can verify that the second term in the above expression of $\varphi$ is equal to $\frac{1}{2^{n}}(2|I|-n+1)$ while the third term is equal to $\frac{1}{2^{n-1}}(2|R \cap I|-|R \cap U|)$. Therefore,

$$
\varphi=\frac{1}{2^{n-1}}(|I|+|R \cap U|-2|R \cap I|) \geq 0
$$

since $I \subseteq U$. By Lemma 2 (ii), this shows that $M_{U}\left(v_{R} * y\right) \succeq 0$.

Finally, $M_{V}(y) \succeq 0$, since $\sum_{I \subseteq H}(-1)^{|H \backslash I|} y_{H}=\frac{1}{2^{n}} \geq 0$.

Example 18. Consider the polytope

$$
K:=\left\{x \in[0,1]^{n} \mid \sum_{i=1}^{n} x_{i} \geq \frac{1}{2}\right\},
$$

then $P=\left\{x \in[0,1]^{n} \mid \sum_{i=1}^{n} x_{i} \geq 1\right\}$. This example was considered by Cook and Dash (2001) as an example where the Lovász-Schrijver rank is $n$. The next result shows that the Sherali-Adams rank is also equal to $n$.

Proposition 19. Let $y \in \mathcal{P}(V)$ with zero entries except $y_{\emptyset}:=1$ and $y_{i}:=\frac{1}{n+1}(i \in V)$. Then, $y \in R_{n-1}(K)$ where $K$ is defined by (26). Therefore, $P \subset S_{n-1}(K)$. 
Proof. One can easily verify (using Lemma 2 (ii)) that $M_{V}(y) \succeq 0$ and $M_{U}(g * y) \succeq 0$ for $U \subseteq V$ with $|U|=n-1$, where $g(x)$ is the polynomial $-\frac{1}{2}+\sum_{i=1}^{n} x_{i}$.

It would be interesting to determine the Lasserre rank of the polytope $K$ in the above two examples. In the second example, when $K$ is defined by (26), we verified that the Lasserre rank is equal to $n$ when $n=2$; indeed, the minimum value of $x_{1}+x_{2}$ for $x \in Q_{1}(K)$ is equal to $\frac{25}{26}<1$. It is not clear how to construct a point $x \in Q_{n-1}(K)$ with $\sum_{i} x_{i}<1$ for general $n \geq 2$.

On the other hand, when $K$ is given by (24), we verified that the Lasserre rank of $K$ is equal to 1 when $n=2$. Again it would be interesting to determine the exact rank for higher values of $n$ (we believe that $n-1$ is the correct value).

\section{Two Applications}

We describe here how the constructions of Lasserre, Lovász-Schrijver, and Sherali-Adams apply to two concrete examples, namely, to the stable set polytope and to the cut polytope of a graph. They are the two most extensively studied examples with respect to this class of methods; the original paper by Lovász and Schrijver (1991) studies the stable set problem while the paper by Laurent (2001) studies the case of max-cut. Moreover, these two examples have been the objects of milestone results in the field of semidefinite optimization.

Indeed, the idea of constructing semidefinite relaxations for a combinatorial problem goes back to the seminal work of Lovász (1979) who introduced the semidefinite bound $\vartheta(G)$ for the stability number of a graph $G$, obtained by optimizing over the semidefinite relaxation $\operatorname{TH}(G)$ (see (27) of the stable set polytope $\mathrm{ST}(G)$ of $G$. An important result is that $\operatorname{TH}(G)=\operatorname{ST}(G)$ precisely when $G$ is a perfect graph, in which case one can solve the maximum stable set problem in polynomial time (with an arbitrary precision) using semidefinite programming; this is still the only polynomial time algorithm known up to today (cf Grötschel, Lovász and Schrijver 1988).

This idea of approximating combinatorial problems using semidefinite relaxations was used later again successfully by Goemans and Williamson (1995) who, using a basic semidefinite relaxation of the cut polytope (defined later in (35)), could prove a good approximation algorithm for the maxcut problem. Since then, semidefinite relaxations have been widely used (in conjonction with clever rounding schemes) for constructing good approximation algorithms for a large number of combinatorial problems. It is therefore of interest to construct new stronger semidefinite relaxations for the stable set polytope and for the cut polytope, as they could potentially be used for designing better approximation algorithms.

\subsection{Application to the stable set polytope}

Given a graph $G=(V=\{1, \ldots, n\}, E)$, let $\mathrm{ST}(G)$ denote the stable set polytope of $G$, let

$$
\operatorname{FR}(G):=\left\{x \in \mathbb{R}_{+}^{n} \mid x_{i}+x_{j} \leq 1 \forall i j \in E\right\}
$$


be its basic linear relaxation defined by nonnegativity and the edge inequalities, and let

$$
\begin{aligned}
\operatorname{TH}(G):=\left\{x \in \mathbb{R}^{n} \mid\right. & \left(\begin{array}{l}
1 \\
x
\end{array}\right)=Y e_{0} \text { for some positive semidefinite matrix } Y=\left(Y_{i j}\right)_{i, j=0}^{n} \\
& \text { satisfying } \left.Y_{i i}=Y_{0 i}(i \in V), Y_{i j}=0(i j \in E)\right\}
\end{aligned}
$$

be the basic semidefinite relaxation of $\mathrm{ST}(G)$. Let us compare how the various methods apply to the pair $P:=\mathrm{ST}(G), K:=\mathrm{FR}(G)$.

Define the $N$-rank (resp., $N_{+}$-rank) of $\operatorname{FR}(G)$ as the smallest integer $t$ for which $N^{t}(\mathrm{FR}(G))=$ $\mathrm{ST}(G)$ (resp., $N_{+}^{t}(\mathrm{FR}(G))=\mathrm{ST}(G)$ ); define similarly the $S A$-rank and the Lasserre rank of $\mathrm{FR}(G)$.

The relaxations $N(\operatorname{FR}(G))$ and $N_{+}(\operatorname{FR}(G))$ are studied in detail in (Lovász and Schrijver 1991). In particular, the following results are shown there. The polytope $N(\operatorname{FR}(G))$ is defined by the nonnegativity and edge constraints together with the odd hole inequalities: $\sum_{i \in V(C)} x_{i} \leq \frac{|C|-1}{2}$ for $C$ odd hole in $G$. If $G$ has $n$ nodes and stability number $\alpha(G)$, then its $N$-rank $t$ satisfies:

$$
\frac{n}{\alpha(G)}-2 \leq t \leq n-\alpha(G)-1
$$

the $N$-rank $t$ of an inequality $a^{T} x \leq \beta$ valid for $\operatorname{ST}(G)$ (with integer coefficients and distinct from the nonnegativity constraints) satisfies:

$$
\frac{1}{\beta}\left(\sum_{i \in V} a_{i}-2 \beta\right) \leq t \leq \sum_{i \in V} a_{i}-2 \beta .
$$

The lower bounds follow from the fact that

$$
\frac{1}{t+2}(1, \ldots, 1)^{T} \in N^{t}(\mathrm{FR}(G))
$$

for any $t \geq 0$. The $N_{+}$operator yields a much stronger relaxation, as clique inequalities, odd wheel and odd antihole inequalities are valid for $N_{+}(\operatorname{FR}(G)$ ) (while the $N$-rank of a clique inequality based on a clique of size $k$ is $k-2$ ). Thus, perfect graphs have $N_{+}$-rank 1 . Moreover,

$$
N_{+}(\operatorname{FR}(G)) \subseteq \operatorname{TH}(G)
$$

for any graph $G$ and the $N_{+} \operatorname{rank} t$ of $G$ satisfies:

$$
t \leq \alpha(G)
$$

The Sherali-Adams hierarchy does not seem to yield a significant improvement with respect to the sequence $N^{t}(\operatorname{FR}(G))$. Indeed, the vector $\frac{1}{t+2}(1, \ldots, 1)^{T} \in \mathbb{R}^{n}$ considered in (30) belongs also to $S_{t}(\operatorname{FR}(G))$. (Because the vector $y \in \mathbb{R}^{\mathcal{P}_{t+1}(V)}$ defined by $y_{\emptyset}:=1, y_{I}:=\frac{1}{t+2}$ if $|I|=1$, and $y_{I}:=0$ if $|I| \geq 2$ belongs to $R_{t}(\operatorname{FR}(G))$.) Therefore, the lower bounds from (28) and (29) remain valid for the SA-rank of $\operatorname{FR}(G)$.

On the other hand, the Lasserre hierarchy does improve on the sequence $N_{+}^{t}(\operatorname{FR}(G))$ as we now see. We begin with giving a more compact formulation for the relaxation $Q_{t}(\operatorname{FR}(G))$. For an edge $a b \in E$, 
let $g_{a b}(x):=1-x_{a}-x_{b}$ be the polynomial corresponding to the edge inequality $x_{a}+x_{b} \leq 1$. We show that the positive semidefinite constraint $M_{t}\left(g_{a b} * y\right) \succeq 0$ can be replaced by the linear equation: $y_{a b}=0$.

Lemma 20. Let $t \geq 1$ and $y \in \mathbb{R}^{\mathcal{P}_{2 t+2}(V)}$. The following assertions are equivalent.

(i) $y \in P_{t}(\mathrm{FR}(G))$

(ii) $M_{t+1}(y) \succeq 0$ and $y_{a b}=0$ for any edge $a b \in E$.

(iii) $M_{t+1}(y) \succeq 0$ and $y_{I}=0$ for any $I \in \mathcal{P}_{2 t+2}(V)$ which is not stable.

Proof. Note first that the condition $M_{t+1}(y) \succeq 0$ implies that $y_{I} \geq 0$ for all $I \in \mathcal{P}_{t+1}(V)$.

(i) $\Longrightarrow$ (ii) The $(a, a)$-th entry of $M_{t}\left(g_{a b} * y\right)$ is equal to $g_{a b} * y(a)=-y_{a b}$ and is nonnegative, which implies that $y_{a b}=0$.

(ii) $\Longrightarrow$ (iii) Suppose $I$ contains the edge $a b$. If $|I| \leq t+1$, then the $(a b, I)$-th entry of $M_{t+1}(y)$ is equal to 0 since the $(a b, a b)$-th entry is 0 , which implies that $y_{I}=0$. Otherwise, write $I=I_{1} \cup I_{2}$ where $I_{1}, I_{2} \in \mathcal{P}_{t+1}(V)$ with $\{a, b\} \subseteq I_{1}$; by the above the $\left(I_{1}, I_{1}\right)$-th entry of $M_{t+1}(y)$ is 0 and thus its $\left(I_{1}, I_{2}\right)$-th entry too is 0 , implying $y_{I}=0$.

(iii) $\Longrightarrow$ (i) We show that $M_{t}\left(g_{a b} * y\right) \succeq 0$. Set $\mathcal{P}_{0}:=\mathcal{P}_{t}(V \backslash\{a, b\})$ and $\mathcal{P}_{c}:=\left\{I \cup\{c\} \mid I \in \mathcal{P}_{0}\right\}$ for $c=a$ or $b$. Then, the principal submatrix $X$ of $M_{t+1}(y)$ indexed by $\mathcal{P}_{0} \cup \mathcal{P}_{a} \cup \mathcal{P}_{b}$ has the form:

$\begin{array}{lll}\mathcal{P}_{0} & \mathcal{P}_{a} & \mathcal{P}_{b}\end{array}$

$\mathcal{P}_{0}$
$\mathcal{P}_{a}$
$\mathcal{P}_{b}$$\left(\begin{array}{ccc}C & A & B \\ A & A & 0 \\ B & 0 & B\end{array}\right)$. The condition $X \succeq 0$ implies that $C-A-B \succeq 0$. (To see it, note that $\left(\begin{array}{lll}-x^{T} & x^{T} & x^{T}\end{array}\right) X\left(\begin{array}{c}-x \\ x \\ x\end{array}\right)=x^{T}(C-A-B) x$ for all $\left.x \in \mathbb{R}^{p}, p:=\left|\mathcal{P}_{0}\right|.\right)$ The result now follows since, with respect to the partition of $\mathcal{P}_{t}(V)$ into $\mathcal{P}_{0}$ and its complement $\mathcal{P}_{0}^{\prime}$, the matrix $M_{t}\left(g_{a b} * y\right)$ has the form:

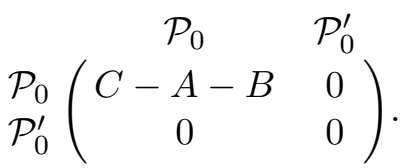

In view of Corollary 15 and (31), it follows that $Q_{\alpha(G)}(\operatorname{FR}(G))=\operatorname{ST}(G)$. In fact, the Lasserre hierarchy already finds $\operatorname{ST}(G)$ at step $\alpha(G)-1$.

Proposition 21. $\mathrm{ST}(G)=Q_{\alpha(G)-1}(\operatorname{FR}(G))$ for a graph $G$ with stability number $\alpha(G) \geq 2$.

Proof. We show that $Q_{\alpha-1}(\operatorname{FR}(G)) \subseteq Q_{n}(\operatorname{FR}(G))$, where $\alpha:=\alpha(G)$. Let $y \in P_{\alpha-1}(\operatorname{FR}(G))$; define $z \in \mathbb{R}^{\mathcal{P}(V)}$ by $z_{I}:=y_{I}$ if $|I| \leq 2 \alpha$ and $z_{I}:=0$ otherwise. Thus, $z_{a b}=0$ for all edges $a b \in E$. By Lemma 20, it suffices to verify that $M_{V}(z) \succeq 0$, which holds since, with respect to the partition of $\mathcal{P}(V)$ into $\mathcal{P}_{\alpha}(V)$ and its complement, $M_{V}(z)$ has the form $\left(\begin{array}{cc}M_{\alpha}(y) & 0 \\ 0 & 0\end{array}\right)$. 
Let $G$ be the line graph of $K_{n}$ with $n$ odd; then, ST $(G)$ is the matching polytope of $K_{n}$. Stephen and Tunçel (1999) show that $\alpha(G)=\frac{n-1}{2}$ iterations of the $N_{+}$operator are needed for finding $\mathrm{ST}(G)$. Therefore, this gives an instance of a graph $G$ for which $\operatorname{ST}(G)=Q_{\alpha-1}(\operatorname{FR}(G))$ is strictly contained in $N_{+}^{\alpha-1}(\mathrm{FR}(G))$.

We conclude with a comparison with the basic semidefinite relaxation $\mathrm{TH}(G)$. By the definition (27), $\operatorname{TH}(G)$ can be seen as the projection on $\mathbb{R}^{n}$ of the set of vectors $y \in \mathbb{R}^{\mathcal{P}_{2}(V)}$ satisfying $y_{\emptyset}=1$ and

$$
M_{1}(y) \succeq 0, y_{a b}=0(a b \in E) .
$$

Therefore, we have the following chain of inclusions:

$$
Q_{1}(\mathrm{FR}(G)) \subseteq N_{+}(\mathrm{FR}(G)) \subseteq \mathrm{TH}(G) \subseteq Q_{0}(\mathrm{FR}(G))
$$

and, in view of Lemma 20, the Lasserre relaxations $Q_{t}(\operatorname{FR}(G))(t \geq 1)$ are natural refinements of the basic SDP relaxation $\mathrm{TH}(G)$.

\subsection{Application to the max-cut problem}

Given a graph $G=(V=\{1, \ldots, n\}, E)$, the max-cut problem asks for a partition $(S, V \backslash S)$ maximizing the total cardinality (or weight) of the edges $i j$ cut by the partition (i.e., such that $|S \cap\{i, j\}|=1$ ). Hence it can be formulated as an unconstrained quadratic \pm 1 -problem:

$$
\max \left(x^{T} A x \mid x \in\{ \pm 1\}^{n}\right),
$$

where $A$ is a (suitably defined) symmetric matrix, but the treatment below remains valid for $A$ arbitrary.

Since we are now working with \pm 1 variables in place of $0-1$ variables, we should adapt some of the definitions given earlier in the paper. In particular, given $K \subseteq[-1,1]^{n}$, the conditions (2) and (3) defining the matrix set $M(K)$ in the Lovász-Schrijver procedure read now: $y_{j j}=y_{00}$ and $Y\left(e_{0} \pm e_{j}\right) \in \tilde{K}$ for $j=1, \ldots, n$. (Indeed, these are the conditions satisfied by matrix $Y:=\left(\begin{array}{l}1 \\ x\end{array}\right)\left(\begin{array}{l}1 \\ x\end{array}\right)^{T}$ for $x \in K \cap\{ \pm 1\}^{n}$.) When defining the moment matrices in (5), one should consider the semigroup $\mathcal{P}(V)$ with the symmetric difference as semigroup operation in place of the union. Namely, the $(I, J)$ th entry of a moment matrix $M_{V}(y)$ is now $y(I \Delta J)$ instead of $y(I \cup J)$. (Indeed, for $x \in \mathbb{R}^{n}$ and $y:=$ $\left(\prod_{i \in I} x_{i}\right)_{I \subseteq V}$, the $(I, J)$-th entry of matrix $y y^{T}$ is equal to $y(I \Delta J)$ when $x$ is \pm 1 -valued, while equal to $y(I \cup J)$ when $x$ is $0-1$ valued.) Moreover, the zeta matrix is now defined as $Z=\left((-1)^{|I \cap J|}\right)_{I, J \subseteq V}$ with inverse $Z^{-1}=2^{-n} Z$.

There are two possible strategies in order to formulate relaxations for the problem (32).

\subsubsection{First strategy}

The first possible strategy is to formulate (32) as a linear problem

$$
\max \left(\langle A, X\rangle \mid X \in \mathrm{CUT}_{n}\right)
$$


over the cut polytope

$$
\mathrm{CUT}_{n}:=\operatorname{conv}\left(x x^{T} \mid x \in\{ \pm 1\}^{n}\right)
$$

(which is in fact a $\left(\begin{array}{l}n \\ 2\end{array}\right)$-dimensional polytope) and to apply the various constructions to some linear programming formulation of $\mathrm{CUT}_{n}$. As linear programming formulation for $\mathrm{CUT}_{n}$, one can take the metric polytope $\mathrm{MET}_{n}$ consisting of the symmetric matrices $X$ with an all ones diagonal and satisfying the triangle inequalities:

$$
X_{i j}+X_{i k}+X_{j k} \geq-1, X_{i j}-X_{i k}-X_{j k} \geq-1
$$

for all distinct $i, j, k \in V$.

One can also consider linear relaxations of the cut polytope $\operatorname{CUT}(G)$ of an arbitrary graph $G$. Given a graph $G=(V, E)$, let $\operatorname{CUT}(G)$ and $\operatorname{MET}(G)$ denote the projections of $\mathrm{CUT}_{n}$ and $\mathrm{MET}_{n}$, respectively, on the subspace $\mathbb{R}^{E}$ indexed by the edge set of $G$. Then, $\operatorname{CUT}(G) \subseteq \operatorname{MET}(G)$ with equality if and only if $G$ has no $K_{5}$-minor (Barahona and Mahjoub 1986).

When applying the Lovász-Schrijver construction to $K:=\operatorname{MET}(G)$, one finds the relaxation $N(\operatorname{MET}(G))$ of $\operatorname{CUT}(G)$. Another possibility is to first apply the LS construction to $K:=\operatorname{MET}\left(K_{n}\right)$ and then project back on the edge space $\mathbb{R}^{E}$, thus yielding the relaxation $N(G):=\pi_{E}\left(N\left(\operatorname{MET}\left(K_{n}\right)\right)\right)$ of $\operatorname{CUT}(G)$. Here, $\pi_{E}$ denotes the projection from the space indexed by the edge set of $K_{n}$ to the space indexed by the edge set of $G$. One has:

$$
N(G) \subseteq N(\operatorname{MET}(G))
$$

but it is not known whether equality holds in general.

The following results about the relaxations $N(G)$ and $N(\operatorname{MET}(G))$ are shown in (Laurent 2001). Equality: $N^{t}(\operatorname{MET}(G))=\operatorname{CUT}(G)$ holds if $G$ has $t$ edges whose contraction produces a graph with no $K_{5}$-minor. In particular, $N^{n-\alpha(G)-3}(G)=\operatorname{CUT}(G)$; moreover, $N^{n-\alpha(G)-3}(\operatorname{MET}(G))=\operatorname{CUT}(G)$ if $G$ has a maximum stable set whose complement induces a graph with at most three connected components. In particular, $N^{n-4}\left(K_{n}\right)=\operatorname{CUT}\left(K_{n}\right)$ for $n \geq 4$. The value $n-4$ is known to be the correct value for the $N$-rank of $\operatorname{MET}\left(K_{n}\right)$ when $n \leq 7$ and is conjectured to be the correct value for any $n$. Although the inclusion $N_{+}(\operatorname{MET}(G)) \subseteq N(\operatorname{MET}(G))$ is strict in general (e.g., for $G=K_{n}$ and $n \geq 6$ ), no example is known of a graph for which the number of iterations needed for finding CUT $(G)$ is smaller when using the $N_{+}$operator instead of the $N$ operator.

When applying the Sherali-Adams and Lasserre constructions to $K=\operatorname{MET}(G)$, one finds the relaxations $S_{t}(\operatorname{MET}(G))$ and $Q_{t}(\operatorname{MET}(G))$ satisfying: $Q_{t}(\operatorname{MET}(G)) \subseteq S_{t}(\operatorname{MET}(G)) \cap N_{+}^{t}(\operatorname{MET}(G))$. The definition of $Q_{t}(\operatorname{MET}(G))$ involves a semidefinite program containing possibly exponentially many constraints (at least as many as the number of circuits in $G$ ). In order to decrease the number of constraints, one can consider instead the set $\pi_{E}\left(Q_{t}\left(\operatorname{MET}\left(K_{n}\right)\right)\right)$ whose definition involves $O\left(n^{3}\right)$ semidefinite constraints. A much simpler relaxation can be obtained by applying the alternative strategy described below.

\subsubsection{Second strategy}

Another possible strategy is to apply the various constructions to the cube $K=C_{n}:=[-1,1]^{n}$ and to take projections on the space $\mathbb{R}^{E_{n}}$ indexed by the set $E_{n}$ of pairs $i j$ of points of $V$ (instead of 
projections on the space $\mathbb{R}^{V}$ indexed by the singletons). Thus we now consider the Sherali-Adams set $R_{t}\left(C_{n}\right)$ and the Lasserre set $P_{t}\left(C_{n}\right)$ and their respective projections $\hat{S}_{t}\left(C_{n}\right)$ and $\hat{Q}_{t}\left(C_{n}\right)$ on $\mathbb{R}^{E_{n}}$. (The 'hat' symbol is meant to remind that the projection is taken over the set of pairs.) We have that

$$
\hat{S}_{n}\left(C_{n}\right)=\hat{Q}_{n-1}\left(C_{n}\right)=\operatorname{CUT}\left(K_{n}\right) .
$$

By the definition, the relaxation $\hat{S}_{t}\left(C_{n}\right)$ consists of the vectors $y \in \mathbb{R}^{E_{n}}$ whose restriction on a subset of $t$ points belongs to $\operatorname{CUT}\left(K_{t}\right)$. In other words, $\hat{S}_{t}\left(C_{n}\right)$ is the polytope in $\mathbb{R}^{E_{n}}$ determined by all the valid inequalities for $\operatorname{CUT}\left(K_{n}\right)$ on at most $t$ points. For instance, $\hat{S}_{2}\left(C_{n}\right)=[-1,1]^{E_{n}}$ while $\hat{S}_{3}\left(C_{n}\right)$ is the metric polytope $\operatorname{MET}\left(K_{n}\right)$.

For $t \geq 0$, the $t$-th Lasserre relaxation of the max-cut problem reads:

$$
\max \left(\sum_{i, j \in V} a_{i j} y_{i j} \mid M_{t+1}(y)=(y(I \Delta J))_{I, J \in \mathcal{P}_{t+1}(V)} \succeq 0, y_{\emptyset}=1\right) .
$$

Let $\tilde{M}_{t+1}(y)$ denote the principal submatrix of $M_{t+1}(y)$ whose rows and columns are indexed by the sets $I \in \mathcal{P}_{t+1}(V)$ whose cardinality has the same parity as $t+1$. In fact, the program (33) can be reformulated as the smaller program:

$$
\max \left(\sum_{i, j \in V} a_{i j} y_{i j} \mid \tilde{M}_{t+1}(y) \succeq 0, y_{\emptyset}=1\right) .
$$

Indeed, write $M_{t+1}(y)=\left(\begin{array}{cc}A & C \\ C^{T} & B\end{array}\right)$, where $A$ is the submatrix of $M_{t+1}(y)$ indexed by all even sets and $B$ its submatrix indexed by all odd sets. As the objective function in (33) does not involve any variable $y_{I}$ with $|I|$ odd, we can assume that $C=0$. Moreover, $A$ is a submatrix of $B$ and $B=\tilde{M}_{t+1}(y)$ if $t+1$ is odd, while $B$ is a submatrix of $A$ and $A=\tilde{M}_{t+1}(y)$ if $t+1$ is even. (To see it, use, e.g., the fact that $I \Delta J=(I \Delta\{1\}) \Delta(J \Delta\{1\})$.) Therefore, the two programs (33) and (34) are equivalent.

Hence we find again the following facts observed by Lasserre (2000). For $t=0$, the feasible set of the program (34) is the basic semidefinite relaxation

$$
\left\{X=\left(x_{i j}\right)_{i, j=1}^{n} \mid X \succeq 0, x_{i i}=1 \forall i=1, \ldots, n\right\} .
$$

For $t=1$, the feasible set of the program (34) is the set $\mathcal{F}_{n}^{\prime}$ consisting of the positive semidefinite matrices $Z$ indexed by $E_{n} \cup\{\emptyset\}$ satisfying

$$
Z_{i j, i k}=Z_{\emptyset, j k} \text { and } Z_{i j, r s}=Z_{i r, j s}=Z_{i s, j r}
$$

for all distinct $i, j, k, r, s \in V$. If we remove in the definition of $\mathcal{F}_{n}^{\prime}$ the condition $Z_{i j, r s}=Z_{i r, j s}=Z_{i s, j r}$, we obtain the larger matrix set $\mathcal{F}_{n}$ underlying the relaxation (SDP3) defined by Anjos and Wolkowicz (2002). Setting

$$
F_{n}:=\left\{x \in \mathbb{R}^{E_{n}} \mid\left(\begin{array}{l}
1 \\
x
\end{array}\right)=Z e_{0} \text { for some } Z \in \mathcal{F}_{n}\right\}
$$

we have:

$$
\operatorname{CUT}\left(K_{n}\right) \subseteq \hat{Q}_{1}\left(C_{n}\right) \subseteq F_{n} \subseteq \hat{Q}_{0}\left(C_{n}\right) \cap \operatorname{MET}\left(K_{n}\right)
$$


The right most inclusion is shown in (Anjos and Wolkowicz 2002); both left and right most inclusions are strict for $n=5$.

Laurent (2002) compares the relaxations $\hat{Q}_{t}\left(C_{n}\right)$ with the Lovász-Schrijver relaxations. In particular, she shows that

$$
\hat{Q}_{t}\left(C_{n}\right) \subseteq N_{+}^{t-1}\left(K_{n}\right) \text { for } t \geq 1 .
$$

Therefore, the set $\hat{Q}_{t}\left(C_{n}\right)$ seems to be the most attractive semidefinite relaxation for the cut polytope, since it is tightest and has a simple direct description, while the set $N_{+}^{t-1}\left(K_{n}\right)$ only has a recursive description.

We conclude with a few observations. It is shown in (Laurent 2001) that $M_{+}\left(\operatorname{MET}\left(K_{n}\right)\right) \subseteq \mathcal{F}_{n}$ and $M_{+}^{\prime}\left(\operatorname{MET}\left(K_{n}\right)\right) \subseteq \mathcal{F}_{n}^{\prime}$. Here, $M_{+}$is the Lovász-Schrijver matrix operator introduced in Section 2 and $M_{+}^{\prime}$ is a strengthening of $M_{+}$considered in (Laurent 2001) (corresponding to the matrix operator $M_{+}(K, K)$ from (Lovász and Schrijver 1991)). Therefore, $N_{+}\left(\operatorname{MET}\left(K_{n}\right)\right) \subseteq F_{n}$ and the operator $M_{+}^{\prime}$ yields a relaxation $N_{+}^{\prime}\left(\operatorname{MET}\left(K_{n}\right)\right)$ which is contained in the Lasserre relaxation $\hat{Q}_{1}\left(C_{n}\right)$. The inclusion $N_{+}^{\prime}\left(\operatorname{MET}\left(K_{n}\right)\right) \subseteq \hat{Q}_{1}\left(C_{n}\right)$ is strict for $n=5$, since $N_{+}^{\prime}\left(\operatorname{MET}\left(K_{5}\right)\right)=\operatorname{CUT}\left(K_{5}\right) \subset \hat{Q}_{1}\left(C_{5}\right)$.

\section{Lasserre's Approach Revisited}

In this section we revisit the hierarchy of relaxations of Lasserre introduced in Section 3 from the algebraic point of view of representing nonnegative polynomials as sums of squares and the dual theory of moments. This approach applies to general (not necessarily $0-1$ ) polynomial programming problems. The idea of approximating polynomial programming problems using sums of squares of polynomials has been used in several other works, in particular, by Shor $(1987,1998)$, Nesterov $(2000)$, Parrilo (2000), De Klerk and Pasechnik (2001). A key result in this approach is the fact that testing whether a polynomial can be written as a sum of squares can be reformulated as a semidefinite programming problem. Based on this (and on the Positivstellensatz in real algebraic geometry), Parrilo (2000) shows that one can test whether a semi-algebraic set is empty using semidefinite programming.

We introduce the main ideas on the unconstrained problem of minimizing a polynomial function over $\mathbb{R}^{n}$; our exposition follows (Lasserre 2001a).

\subsection{A gentle introduction}

Suppose we want to solve the problem:

$$
p^{*}:=\min g(x) \text { subject to } x \in \mathbb{R}^{n},
$$

where $g(x)$ is a polynomial of even degree $2 d$ which can be assumed without loss of generality to satisfy $g(0)=0$. It is easy to see that (36) can be reformulated as

$$
p^{*}=\min _{\mu} \int g(x) d \mu(x)
$$

where the minimum is taken over all probability measures $\mu$ on $\mathbb{R}^{n}$. Write the polynomial $g(x)$ as a sum of monomials: $g(x)=\sum_{\alpha \in \mathcal{S}_{2 d}} g_{\alpha} x^{\alpha}$, where $x^{\alpha}:=x_{1}^{\alpha_{1}} \ldots x_{n}^{\alpha_{n}}$ and, for an integer $m, \mathcal{S}_{m}$ denotes 
the set of sequences $\alpha \in \mathbb{Z}_{+}^{n}$ with $|\alpha|:=\sum_{i=1}^{n} \alpha_{i} \leq m$. Then, $\int g(x) d \mu(x)=\sum_{\alpha} g_{\alpha} \int x^{\alpha} d \mu(x)$. A sequence $y=\left(y_{\alpha}\right)_{\alpha \in \mathcal{S}_{2 d}}$ is said to be a moment sequence if

$$
y_{\alpha}=\int x^{\alpha} d \mu(x)
$$

(for all $\alpha \in \mathcal{S}_{2 d}$ ) for some nonnegative measure $\mu$ on $\mathbb{R}^{n}$. Then (37) can be rewritten as

$$
p^{*}=\min \sum_{\alpha} g_{\alpha} y_{\alpha} \quad \text { s.t. } y \text { is a moment sequence and } y_{0}=1 .
$$

Lower bounds for $p^{*}$ can be obtained by relaxing the condition that $y$ be a moment sequence. A necessary condition for $y$ to be a moment sequence is that its moment matrix

$$
M_{d}^{\mathbb{Z}}(y):=\left(y_{\alpha+\beta}\right)_{\alpha, \beta \in \mathcal{S}_{d}}
$$

be positive semidefinite. Indeed, if $y$ is the moment sequence of a nonnegative measure $\mu$, then

$$
f^{T} M_{d}^{\mathbb{Z}}(y) f=\sum_{\alpha, \beta \in \mathcal{S}_{d}} f_{\alpha} f_{\beta} y_{\alpha+\beta}=\sum_{\alpha, \beta \in \mathcal{S}_{d}} f_{\alpha} f_{\beta} \int x^{\alpha+\beta} d \mu(x)=\int\left(\sum_{\alpha} f_{\alpha} x^{\alpha}\right)^{2} d \mu(x) \geq 0
$$

for all $f=\left(f_{\alpha}\right)_{\alpha \in \mathcal{S}_{d}}$. Write

$$
M_{d}^{\mathbb{Z}}(y)=\sum_{\gamma \in \mathcal{S}_{2 d}} y_{\gamma} B_{\gamma}
$$

where $B_{\gamma}:=\sum_{\alpha, \beta \in \mathcal{S}_{d} \mid \alpha+\beta=\gamma} E_{\alpha, \beta}$ (where $E_{\alpha, \beta}$ denotes the elementary matrix, with all zero entries except ones at the positions $(\alpha, \beta)$ and $(\beta, \alpha))$. Therefore, one has the following lower bound for $p^{*}$ :

$$
\begin{aligned}
p^{*} \geq \min & g^{T} y \\
\text { s.t. } & M_{d}^{\mathbb{Z}}(y) \succeq 0 \\
& y_{0}=1
\end{aligned}
$$

One can also proceed in the following dual manner for computing $p^{*}$. Rewrite (36) as

$$
p^{*}=\max \lambda \text { subject to } g(x)-\lambda \geq 0 \forall x \in \mathbb{R}^{n} \text {. }
$$

Lower bounds for $p^{*}$ can now be obtained by considering sufficient conditions for the polynomial $g(x)-\lambda$ to be nonnegative over $\mathbb{R}^{n}$. An obvious sufficient condition being that $g(x)-\lambda$ be a sum of squares of polynomials. Testing whether a polynomial $p(x)$ is a a sum of squares amounts to deciding feasibility of a semidefinite program (see, e.g., Nesterov (2000), Parrilo (2000)). Indeed, say $p(x)$ has degree $2 d$, and let $z:=\left(x^{\alpha}\right)_{\alpha \in \mathcal{S}_{d}}$ be the vector consisting of all monomials of degree $\leq d$. Then one can easily verify that $p(x)$ is a sum of squares if and only if $p(x)=z^{T} X z$ (identical polynomials) for some positive semidefinite matrix $X$. As

$$
z^{T} X z=\sum_{\alpha, \beta \in \mathcal{S}_{d}} X_{\alpha, \beta} x^{\alpha+\beta}=\sum_{\gamma \in \mathcal{S}_{2 d}} x^{\gamma}\left(\sum_{\substack{\alpha, \beta \in \mathcal{S}_{d} \\ \alpha+\beta=\gamma}} X_{\alpha, \beta}\right)=\sum_{\gamma \in \mathcal{S}_{2 d}} x^{\gamma}\left\langle B_{\gamma}, X\right\rangle
$$


it follows that $p(x)$ is a sum of squares if and only if the following SDP program:

$$
X \succeq 0,\left\langle B_{\gamma}, X\right\rangle=p_{\gamma}\left(\gamma \in \mathcal{S}_{2 d}\right)
$$

is feasible, where $X$ is of order $\left(\begin{array}{c}n+d \\ d\end{array}\right)$ and with $\left(\begin{array}{c}n+2 d \\ 2 d\end{array}\right)$ equations (thus polynomially solvable for fixed $n$ or $d$ ). Based on this we can formulate the following lower bound for $p^{*}$ :

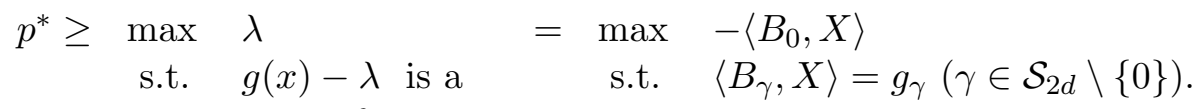

$$
\begin{aligned}
& \text { sum of squares }
\end{aligned}
$$

The SDP programs (42) and (45) are, in fact, dual of each other and there is no duality gap if (45) is feasible.

The lower bound from (45) is equal to $p^{*}$ if $g(x)-p^{*}$ is a sum of squares; this holds for $n=1$ but not in general if $n \geq 2$. In general one can estimate $p^{*}$ asymptotically by a sequence of SDP's analogue to (45) if one assumes that an upper bound $R$ is known a priori on the norm of a global minimizer $x$ of $g(x)$, in which case (36) is equal to

$$
\min g(x) \text { subject to } R-\sum_{i=1}^{n} x_{i}^{2} \geq 0 \text {. }
$$

Using a result of Putinar (cf. Theorem 22 below), it follows that, for any $\epsilon>0, g(x)-p^{*}+\epsilon$ can be decomposed as $p(x)+q(x)\left(R-\sum_{i} x_{i}^{2}\right)$ for some polynomials $p(x)$ and $q(x)$ that are sums of squares. Testing for the existence of such a decomposition can be expressed as a SDP program analogue to (45). Details are given in Section 7.3 where the general problem of minimizing a polynomial function over a semi-algebraic set is considered. Section 7.2 contains preliminaries over moment sequences and polynomials.

\subsection{The moment problem and sums of squares of polynomials}

\subsubsection{The moment problem}

Let $(S,+)$ be a commutative semigroup and let $S^{*}$ denote the set of nonzero mappings $f: S \longrightarrow \mathbb{R}$ that are multiplicative, i.e., satisfy $f(\alpha+\beta)=f(\alpha) f(\beta)$ for all $\alpha, \beta \in S$. Given a sequence $y=\left(y_{\alpha}\right)_{\alpha \in S}$ indexed by $S$, its moment matrix $M(y)$ is the $S \times S$ matrix whose $(\alpha, \beta)$-th entry is $y_{\alpha+\beta}$ for $\alpha, \beta \in S$.

When $S$ is the semigroup $\mathcal{P}(V)$ with the union as semigroup operation, we find the moment matrix $M_{V}(y)$ already introduced earlier in (5). When $S$ is the semigroup $\left(\mathbb{Z}_{+}^{n},+\right)$, we use the notation $M^{\mathbb{Z}}(y)$ for the moment matrix of $y \in \mathbb{R}^{\mathbb{Z}_{+}^{n}}$ and $M_{t}^{\mathbb{Z}}(y)$ for its principal submatrix indexed by all sequences $\alpha \in \mathbb{Z}_{+}^{n}$ with $|\alpha| \leq t$, already considered earlier in (40).

Following (Berg, Christensen and Jensen 1979, Berg, Christensen and Ressel 1984), a sequence $y \in \mathbb{R}^{S}$ is said to be positive semidefinite if every finite principal submatrix of its moment matrix $M(y)$ is positive semidefinite. Given a subset $F \subseteq S^{*}$, a sequence $y \in \mathbb{R}^{S}$ is called a $F$-moment sequence if there exists a positive Radon measure $\mu$ on $S^{*}$ supported by $F$ such that

$$
y_{\alpha}=\int_{S^{*}} f_{\alpha} d \mu(f) \text { for all } \alpha \in S .
$$


Given two sequences $x, y \in \mathbb{R}^{S}$, definition (6) extends as

$$
(x * y)_{\alpha}:=\sum_{\gamma \in S} x_{\gamma} y_{\alpha+\gamma} \text { for } \alpha \in S .
$$

The moment problem is the problem of characterizing moment sequences. It has been much studied in the literature especially for the semigroup $S=\mathbb{Z}_{+}^{n}$, in which case $S^{*}=\mathbb{R}^{n}$ and the moment condition (46) reads as relation (38); see (Fuglede 1983, Berg, Christensen and Ressel 1984) for a survey.

Obviously, every $F$-moment sequence should be positive semidefinite. Much research has been done for characterizing moment sequences for various closed sets $F$. For instance, for $n=1$ and $F=\mathbb{R}$, every positive semidefinite sequence is a moment sequence, a result of Hamburger in 1920 . For $n=1$ and $F=\mathbb{R}_{+}$, a sequence $y=\left(y_{i}\right)_{i \geq 0}$ is a $F$-moment sequence if and only if both $y$ and $e_{1} * y=\left(y_{i+1}\right)_{i \geq 0}$ are positive semidefinite, a result shown by Stieltjes in 1894. For $n=1$ and $F=[0,1]$, a sequence $y=\left(y_{i}\right)_{i \geq 0}$ is a $F$-moment sequence (then called a Hausdorff sequence) if and only if $e_{1} * y$ and $y-e_{1} * y$ are positive semidefinite. When $F$ is a compact semi-algebraic set in $\mathbb{R}^{n}$ of the form

$$
F=\left\{x \in \mathbb{R}^{n} \mid g_{\ell}(x) \geq 0 \text { for } \ell=1, \ldots, m\right\}
$$

where $g_{\ell}$ are polynomials, Schmüdgen (1991) shows that $y$ is a $F$-moment sequence if and only if $y$ and $g * y$ are positive semidefinite for every product $g=\prod_{\ell \in L} g_{\ell}$ for $L \subseteq\{1, \ldots, m\}$.

\subsubsection{Reformulating Corollary 3 as a moment result}

In fact, the result from Corollary 3 can also be viewed as a result about moments, if we consider sequences indexed by the semigroup $S:=\mathcal{P}(V)$ with the union as semigroup operation. Then, $S^{*}=\left\{\zeta^{S} \mid S \in \mathcal{P}(V)\right\}$. Hence, a sequence $y \in \mathbb{R}^{\mathcal{P}(V)}$ is a moment sequence if and only if $y \in \mathcal{C}_{\mathcal{P}(V)}$ which, by Corollary 3 , is equivalent to $y$ being a positive definite sequence. (Noting that $\mathcal{P}(V)$ is an idempotent semigroup, this result also follows from Proposition 4.17 in (Berg, Christensen and Ressel 1984).)

More generally, let $F=\left\{x \in\{0,1\}^{n} \mid g_{\ell}(x) \geq 0 \forall l=1, \ldots, m\right\}$, where the $g_{\ell}$ 's are polynomials in which each variable occurs with degree $\leq 1$, and let $\mathcal{J}$ be defined as in (14). Then, $y$ is a $F$-moment sequence (meaning that the measure $\mu$ is nonzero only at $\zeta^{S}$ with $\chi^{S} \in F$, i.e., $S \in \mathcal{J}$ ) if and only if $y \in \mathcal{C}_{\mathcal{J}}$. By Corollary 3 , this is equivalent to the sequences $y$ and $g_{\ell} * y(\ell=1, \ldots, m)$ being positive semidefinite. Therefore, this gives a 'discrete' analogue of the above mentioned result of Schmüdgen.

\subsubsection{Representations of nonnegative polynomials as sums of squares}

Let $\mathcal{P}_{+}(F)$ denote the set of polynomials $p(x)=\sum_{\alpha} p_{\alpha} x^{\alpha}$ that are nonnegative on $F$; that is, $p(x) \geq 0$ for all $x \in F$. One of the basic results about moments (Haviland 1935, 1936) is that, given a closed subset $F$ in $\mathbb{R}^{n}, y=\left(y_{\alpha}\right)_{\alpha \in \mathbb{Z}_{+}^{n}}$ is a $F$-moment sequence if and only if $y^{T} p \geq 0$ for every polynomial $p=\left(p_{\alpha}\right)_{\alpha \in \mathbb{Z}_{+}^{n}}$ in $\mathcal{P}_{+}(F)$.

Since a linear functional $f$ on the set $\mathbb{R}\left[x_{1}, \ldots, x_{n}\right]$ of polynomials is completely determined by

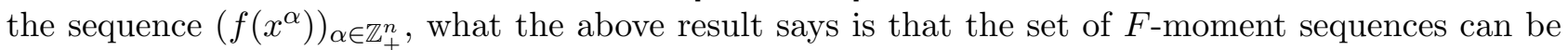
identified with the set of linear functionals that are nonnegative on $\mathcal{P}_{+}(F)$. 
Let $\Sigma^{2}$ denote the convex cone generated by all squares of polynomials in $\mathbb{R}\left[x_{1}, \ldots, x_{n}\right]$. One can easily verify that a linear functional $f$ on $\mathbb{R}\left[x_{1}, \ldots, x_{n}\right]$ is nonnegative on $\Sigma^{2}$ if and only if the sequence $\left(f\left(x^{\alpha}\right)\right)_{\alpha \in \mathbb{Z}_{+}^{n}}$ is positive semidefinite. The obvious inclusion

$$
\Sigma^{2} \subseteq \mathcal{P}_{+}(F)
$$

corresponds by duality to the fact that every $F$-moment sequence is positive semidefinite. For $n=$ $1, F=\mathbb{R}$, it is well known that every nonnegative polynomial on $\mathbb{R}$ can be represented as the sum of squares of two polynomials, which gives again the result of Hamburger. For $n \geq 2$, not every nongegative polynomial can be expressed as a sum of squares of polynomials. This problem of representing polynomials as sums of squares goes back to Hilbert's 17th problem; see (Reznick 1998) for a survey.

Let us reformulate the result of Schmüdgen in terms of polynomials. Let $F$ be as in (47) and let

$$
\Sigma^{2}\left(g_{1}, \ldots, g_{m}\right):=\sum_{I \subseteq\{1, \ldots, m\}}\left(\prod_{i \in I} g_{i}\right) \Sigma^{2}
$$

denote the set of all polynomials of the form $\sum_{I \subseteq\{1, \ldots, m\}} p_{I} \cdot \prod_{i \in I} g_{i}$, where all $p_{I}$ belong to $\Sigma^{2}$. One can easily verify that a linear functional $f$ on $\mathbb{R}\left[x_{1}, \ldots, x_{n}\right]$ is nonnegative on $\Sigma^{2}\left(g_{1}, \ldots, g_{m}\right)$ if and only if the associated sequence $y:=\left(f\left(x^{\alpha}\right)\right)_{\alpha}$ is positive semidefinite as well as the sequences $\left(\prod_{i \in I} g_{i}\right) * y$ for all $I \subseteq\{1, \ldots, m\}$. Therefore, what Schmüdgen shows is that both sets $\mathcal{P}_{+}(F)$ and $\Sigma^{2}\left(g_{1}, \ldots, g_{m}\right)$ have the same sets of nonnegative linear functionals. From this follows that every polynomial $p$ which is positive on $F$ belongs to $\Sigma^{2}\left(g_{1}, \ldots, g_{m}\right)$. Putinar (1993) shows the following stronger result which, as we will see in the next subsection, plays a central role in the approach of Lasserre for asymptotically evaluating polynomial programs.

Theorem 22. (Putinar 1993) Let $F$ be a compact semi-algebraic set as in (47). Assume that there exists a polynomial $u \in \Sigma^{2}+g_{1} \Sigma^{2}+\ldots+g_{m} \Sigma^{2}$ for which the set $\left\{x \in \mathbb{R}^{n} \mid u(x) \geq 0\right\}$ is compact. If $p$ is a polynomial positive on $F$, then $p \in \Sigma^{2}+g_{1} \Sigma^{2}+\ldots g_{m} \Sigma^{2}$.

\subsection{Lasserre's method for polynomial programs}

\subsubsection{Successive relaxations for polynomial programs}

Let $F$ be a semi-algebraic set as in (47). Assume that the assumptions from Theorem 22 hold; that is, $F$ is compact and the set $\left\{x \in \mathbb{R}^{n} \mid u(x) \geq 0\right\}$ is compact for some polynomial $u \in \Sigma^{2}+g_{1} \Sigma^{2}+\ldots g_{m} \Sigma^{2}$. (This is true, for instance, when the description of $F$ includes a constraint $g_{\ell}(x) \geq 0$ for which the set $\left\{x \mid g_{\ell}(x) \geq 0\right\}$ is compact, or when the constraints $x_{i}^{2}-x_{i}=0$ are present in the description of $F$ (the $0-1$ case).) Suppose we want to solve the problem

$$
p^{*}:=\min g_{0}(x) \text { subject to } x \in F
$$


where $g_{0}$ is a polynomial of degree $w_{0}$ which can be assumed to satisfy $g_{0}(0)=0$. Let $w_{\ell}$ denote the degree of $g_{\ell}, v_{\ell}:=\left\lceil\frac{w_{\ell}}{2}\right\rceil, v:=\max _{\ell=1, \ldots, m} v_{\ell}$ as in (15).

Lasserre (2001a) constructs successive relaxations for problem (48) that converge asymptotically to its optimum solution. His construction is based on the following observation. For $x \in \mathbb{R}^{n}$, define the vector $y^{x} \in \mathbb{R}^{\mathbb{Z}_{+}^{n}}$ with $\alpha$-th entry $x^{\alpha}$ for $\alpha \in \mathbb{Z}_{+}^{n}$. Then, $M^{\mathbb{Z}}\left(y^{x}\right)=y^{x}\left(y^{x}\right)^{T} \succeq 0$ and $M^{\mathbb{Z}}\left(g * y^{x}\right)=$ $g(x) \cdot y^{x}\left(y^{x}\right)^{T} \succeq 0$ if $g(x) \geq 0$. This leads to the following semidefinite relaxation of problem (48) for any $t \geq \max \left(v_{0}-1, v-1\right)$ :

$$
\begin{aligned}
p_{t}^{*}:=\min & \sum_{\alpha}\left(g_{0}\right)_{\alpha} y_{\alpha} \\
\text { s.t. } & M_{t+1}^{\mathbb{Z}}(y) \succeq 0 \\
& M_{t-v_{\ell}+1}^{\mathbb{Z}}\left(g_{\ell} * y\right) \succeq 0(\ell=1, \ldots, m) \\
& y_{0}=1
\end{aligned}
$$

The dual SDP program of (49) reads:

$$
\begin{aligned}
\rho_{t}^{*}:=\max & -X(0,0)-\sum_{\ell=1}^{m} g_{\ell}(0) Z_{\ell}(0,0) \\
\text { s.t. } & \left\langle X, B_{\gamma}\right\rangle+\sum_{\ell=1}^{m}\left\langle Z_{\ell}, C_{\gamma}^{\ell}\right\rangle=\left(g_{0}\right)_{\gamma}(\gamma \neq 0) \\
& X, Z_{\ell} \succeq 0 \stackrel{(\ell=1, \ldots, m),}{ }
\end{aligned}
$$

where $M_{t+1}^{\mathbb{Z}}(y)=\sum_{\gamma} y_{\gamma} B_{\gamma}($ as in (41), with $d=t+1)$ and $M_{t-v_{\ell}+1}^{\mathbb{Z}}\left(g_{\ell} * y\right)=\sum_{\gamma} y_{\gamma} C_{\gamma}^{\ell}$, with $C_{\gamma}^{\ell}=$ $\sum_{\substack{\alpha, \beta \in \mathcal{S}_{t-v_{\ell}+1}, \delta \\ \alpha+\beta+\delta=\gamma}}\left(g_{\ell}\right)_{\delta} E_{\alpha, \beta}$. We have:

$$
\rho_{t}^{*} \leq p_{t}^{*} \leq p^{*} .
$$

For $x \in F$, the sequence $y^{x}$ is obviously an $F$-moment sequence (of the Dirac measure at $x$ ) and thus the primal program (49) states necessary conditions for $y$ to be a moment sequence. The dual program (50) is related to representations of positive polynomials on $F$ as the next lemma shows.

Lemma 23. (Lasserre 2001a) The optimum value $\rho_{t}^{*}$ of $(50)$ is equal to the maximum value of a scalar $\rho$ for which the polynomial $g_{0}(x)-\rho$ can be decomposed as

$$
g_{0}(x)-\rho=p_{0}(x)+\sum_{\ell=1}^{m} p_{\ell}(x) g_{\ell}(x)
$$

where $p_{0}(x)$ and $p_{\ell}(x)$ are sums of squares with $\operatorname{deg}\left(p_{0}\right) \leq 2(t+1)$ and $\operatorname{deg}\left(p_{\ell}\right) \leq 2\left(t+1-v_{\ell}\right)$.

Proof. Let $X, Z_{\ell}$ be feasible solutions for (50) with objective value $\rho$. We show that the polynomial $g_{0}(x)-\rho$ has a decomposition as in (51). For this, write

$$
X=\sum_{j=1}^{r_{0}} q_{j} q_{j}^{T}, Z_{\ell}=\sum_{j=1}^{r_{\ell}} q_{\ell j} q_{\ell j}^{T}
$$


for some vectors $q_{j}$ (indexed by $\alpha \in \mathbb{Z}_{+}^{n}$ with $|\alpha| \leq t+1$ ) and $q_{\ell j}$ (indexed by $\alpha \in \mathbb{Z}_{+}^{n}$ with $|\alpha| \leq$ $\left.t+1-v_{\ell}\right)$. Then, the polynomial $g_{0}(x)-\rho$ is equal to

$$
\begin{aligned}
& \sum_{\gamma \neq 0}\left(g_{0}\right)_{\gamma} x^{\gamma}+X(0,0)+\sum_{\ell} g_{\ell}(0) Z_{\ell}(0,0)=\left\langle X, \sum_{\gamma} x^{\gamma} B_{\gamma}\right\rangle+\sum_{\ell}\left\langle Z_{\ell}, \sum_{\gamma} x^{\gamma} C_{\gamma}^{\ell}\right\rangle \\
& =\left\langle X, M_{t+1}^{\mathbb{Z}}\left(y^{x}\right)\right\rangle+\sum_{\ell}\left\langle Z_{\ell}, M_{t-v_{\ell}+1}^{\mathbb{Z}}\left(g_{\ell} * y^{x}\right)\right\rangle=\sum_{j=1}^{r_{0}}\left(q_{j}(x)\right)^{2}+\sum_{\ell} g_{\ell}(x) \cdot\left(\sum_{j=1}^{r_{\ell}}\left(q_{\ell j}(x)\right)^{2}\right),
\end{aligned}
$$

using the facts that $\left\langle X, M_{t+1}^{\mathbb{Z}}\left(y^{x}\right)\right\rangle=\sum_{j} q_{j}^{T} M^{\mathbb{Z}}\left(y^{x}\right) q_{j}=\sum_{j} \sum_{\alpha, \beta} q_{j}(\alpha) q_{j}(\beta) x^{\alpha+\beta}=\sum_{j}\left(q_{j}(x)\right)^{2}$ and $\left\langle Z_{\ell}, M_{t-v_{\ell}+1}^{\mathbb{Z}}\left(g_{\ell} * y^{x}\right)\right\rangle=\sum_{j} g_{\ell}(x)\left(q_{\ell j}(x)\right)^{2}$. Therefore, $g_{0}(x)-\rho$ has a decomposition (51) where $p_{0}(x):=\sum_{j}\left(q_{j}(x)\right)^{2}$ has degree $\leq 2(t+1)$ and $p_{\ell}(x):=\sum_{j}\left(q_{\ell j}(x)\right)^{2}$ has degree $\leq 2\left(t+1-v_{\ell}\right)$.

The above arguments can be reversed to construct from a decomposition of $g_{0}(x)-\rho$ as in (51) with the degree requirements a feasible solution $X, Z_{\ell}$ to (50) with objective value $\rho$.

One can show the asymptotic convergence of the programs (49) and (50) to the optimum value $p^{*}$ of (48). Indeed, for any $\epsilon>0$, the polynomial $g_{0}(x)-p^{*}+\epsilon$ is positive on the set $F$. Therefore, by Theorem 22, the polynomial $g_{0}(x)-p^{*}+\epsilon$ has a decomposition (51) where $p_{0}, p_{\ell}$ are sums of squares with $\operatorname{deg}\left(p_{0}\right) \leq 2(t+1)$ and $\operatorname{deg}\left(p_{\ell}\right) \leq 2\left(t+1-v_{\ell}\right)$ for some integer $t$. By Lemma 23, this implies that $\rho_{t}^{*} \geq p^{*}-\epsilon$.

Therefore, for any $\epsilon>0$, there exists $t$ for which $p^{*}-\epsilon \leq \rho_{t}^{*} \leq p_{t}^{*} \leq p^{*}$. This shows that $\lim _{t \longrightarrow \infty} p_{t}^{*}=p^{*}$ and

$$
p^{*}=\rho_{t}^{*} \text { for some } t \Longleftrightarrow g_{0}(x)-p^{*} \in \Sigma^{2}+\sum_{\ell=1}^{m} g_{\ell} \Sigma^{2}
$$

Moreover,

$$
\operatorname{conv}(F)=\bigcap_{t \geq v-1} \mathcal{Q}_{t}(F)
$$

where $\mathcal{Q}_{t}(F)$ is defined as the projection of the feasible set of the program (49) intersected with the hyperplane $y_{0}=1$, on the subspace $\mathbb{R}^{n}$ indexed by the sequences $\alpha \in \mathbb{Z}_{+}^{n}$ with $|\alpha|=1$.

\subsubsection{Relation with the previously defined Lasserre relaxations for $0-1$ programs}

Consider now the case when $F$ is the set of $0-1$ solutions of a polynomial system; that is,

$$
F=\left\{x \in \mathbb{R}^{n} \mid g_{\ell}(x) \geq 0(\ell=1, \ldots, m), h_{i}(x)=0(i=1, \ldots, n)\right\}
$$

setting $h_{i}(x):=x_{i}-x_{i}^{2}$ for $i=1, \ldots, n$. Then, one can assume without loss of generality that each $g_{\ell}$ has degree at most 1 in every variable and the assumptions from Theorem 22 hold (with $\left.u(x):=\sum_{i=1}^{n} h_{i}(x)\right)$. Using a result of Curto and Fialkow (2000) about rank extensions of moment matrices, Lasserre (2001b) shows finite convergence of the successive relaxations $\mathcal{Q}_{t}(F)$ to $\operatorname{conv}(F)$; namely,

$$
\mathcal{Q}_{n+v-1}(F)=\operatorname{conv}(F) \text {. }
$$


This can also be seen in the following direct way. For this, consider the relaxation

$$
K:=\left\{x \in[0,1]^{n} \mid g_{\ell}(x) \geq 0(\ell=1, \ldots, m)\right\}
$$

of $F$. As we see in Proposition 24 below, the relaxation $\mathcal{Q}_{t}(F)$ coincides with the relaxation $Q_{t}(K)$ introduced earlier in Section 3.3. Proposition 24 shows in fact the following results: Our presentation in Section 3.3 of the Lasserre relaxations in terms of moment matrices indexed by subsets is equivalent to the original definition of Lasserre (in terms of moment matrices indexed by integer sequences); as an application, this gives an elementary proof for the convergence result from relation (53).

Proposition 24. Let $F$ and $K$ be defined by (52), (54) respectively. Then, $\mathcal{Q}_{t}(F)=Q_{t}(K)$ for any $t \geq v-1$ and $\mathcal{Q}_{t}(F)=\mathcal{Q}_{n+v-1}(F)$ for any $t \geq n+v-1$.

Proof. For $\alpha \in \mathbb{Z}_{+}^{n}$, define $\bar{\alpha} \in\{0,1\}^{n}$ by $\bar{\alpha}_{i}:=1$ if and only if $\alpha_{i} \geq 2$. Then, the condition $M_{t}^{\mathbb{Z}}\left(h_{i} * y\right)=0$ means that

$$
y_{\alpha}=y_{\bar{\alpha}}
$$

for any $\alpha$ with $|\alpha| \leq 2 t$. From this follows that the $\alpha$-th column of the moment matrix $M^{\mathbb{Z}}(y)$ is identical to its $\bar{\alpha}$-th column; similarly for the matrices $M^{\mathbb{Z}}\left(g_{\ell} * y\right)$. A first consequence is that, for $t \geq n$,

$$
M_{t}^{\mathbb{Z}}(y) \succeq 0 \Longleftrightarrow M_{n}^{\mathbb{Z}}(y) \succeq 0, \text { and } M_{t}^{\mathbb{Z}}\left(g_{\ell} * y\right) \succeq 0 \Longleftrightarrow M_{n}^{\mathbb{Z}}\left(g_{\ell} * y\right) \succeq 0 .
$$

This shows equality $\mathcal{Q}_{t}(F)=\mathcal{Q}_{n+v-1}(F)$ for $t \geq n+v-1$. Define $z \in \mathbb{R}^{\mathcal{P}(V)}$ with $I$-th entry $z_{I}:=y_{\alpha}$ where $\alpha:=\chi^{I}$, for $I \subseteq V$. As a consequence of $(55), M_{t}(z)$ is a principal submatrix of $M_{t}^{\mathbb{Z}}(y)$ and

$$
M_{t}(z) \succeq 0 \Longleftrightarrow M_{t}^{\mathbb{Z}}(y) \succeq 0 ;
$$

similarly, $M_{t}\left(g_{\ell} * z\right) \succeq 0 \Longleftrightarrow M_{t}^{\mathbb{Z}}\left(g_{\ell} * y\right) \succeq 0$. This shows equality $Q_{t}(K)=\mathcal{Q}_{t}(F)$ for $t \geq v-1$.

\subsubsection{A dual comparison of the Lasserre and Sherali-Adams approaches}

Consider again problem (48) where $F$ is a semi-algebraic set as in (47). Lasserre (2002) investigates how his method for constructing successive semidefinite approximations of (48) relates to the reformulationlinearization technique of Sherali-Adams for constructing linear approximations of (48), from the dual point of view of representing nonnegative polynomials as sums of squares. His work is in some sense complementary to ours, since the present paper aims at comparing the two methods from the primal point of view of giving necessary conditions for moment sequences (our paper is, however, restricted to the case of $0-1$ polynomial programming). Let us briefly mention some of the results of (Lasserre 2002).

The reformulation-linearization technique of Sherali and Adams $(1990,1994)$ was extended to general polynomial programs as (48) (see, e.g., (Sherali and Tuncbilek 1992,1997)). The basic idea for constructing successive linear approximations is to consider the LP relaxation obtained from the linearization of all possible products $\left(g_{1}(x)\right)^{\beta_{1}} \ldots\left(g_{m}(x)\right)^{\beta_{m}} \geq 0$ of the constraints defining $F$, for $\beta \in \mathbb{Z}_{+}^{m}$ 
with $|\beta| \leq t$, for given integer $t$. Denote by $\sigma_{t}^{*}$ the minimum value of $\sum_{\alpha}\left(g_{0}\right)_{\alpha} y_{\alpha}$ subject to $y$ belonging to the above LP relaxation. Therefore, $\sigma_{t}^{*} \leq p^{*}$. Using LP-duality, it is not hard to see that $\sigma_{t}^{*}$ is equal to the maximum value of a scalar $\rho$ for which the polynomial $g_{0}(x)-\rho$ can be decomposed as

$$
g_{0}(x)-\rho=\sum_{\beta \in \mathbb{Z}_{+}^{m},|\beta| \leq t} \lambda_{\beta}\left(g_{1}(x)\right)^{\beta_{1}} \ldots\left(g_{m}(x)\right)^{\beta_{m}}
$$

for some nonnegative scalars $\lambda_{\beta}$. Comparing with Lemma 23, we see that the SDP and LP approaches (in fact, their dual formulations) aim at representing the polynomial $g_{0}(x)-\rho$ in the following two different ways: either as a sum of the $g_{\ell}$ 's weighted by sums of squares (as in (51), in the SDP case), or as a sum of products $g_{1}^{\beta_{1}} \ldots g_{m}^{\beta_{m}}$ weighted by nonnegative scalars (as in (56), in the LP case). For the primal formulations, this corresponds to considering different necessary conditions for moment sequences; namely, requiring that $M_{t+1}(y)$ and $M_{t+1-v_{\ell}}\left(g_{\ell} * y\right)$ be positive semidefinite (in the SDP case), or requiring some of the Hausdorff moment conditions (in the LP case) (see (Lasserre 2002) for details).

By Putinar's result (Theorem 22), a representation of $g_{0}(x)-p^{*}+\epsilon$ as a sum of the $g_{\ell}$ 's weighted by sums of squares always exists for any $\epsilon>0$. This permits to show asymptotic convergence of the SDP bounds $\rho_{t}^{*}$ to $p^{*}$. On the other hand, a representation of $g_{0}(x)-p^{*}+\epsilon$ as a sum of products $g_{1}^{\beta_{1}} \ldots g_{m}^{\beta_{m}}$ weighted by nonnegative scalars is guaranteed to exist only in the linear case, i.e., when $F$ is a polytope (this is a result of Handelman (1988)), in which case there is also asymptotic convergence of the linear bounds $\sigma_{t}^{*}$ to $p^{*}$.

\subsubsection{The quadratic case}

Finally, let us consider here the special case when $F$ is a semi-algebraic set defined by a set of quadratic constraints; that is, each $g_{\ell}$ is of the form $g_{\ell}(x)=x^{T} Q_{\ell} x+2 q_{\ell}^{T} x+\gamma_{\ell}\left(Q_{\ell}\right.$ symmetric $n \times n$ matrix, $\left.q_{\ell} \in \mathbb{R}^{n}, \gamma_{\ell} \in \mathbb{R}\right)$. For $\ell=1, \ldots, m$, set $P_{\ell}:=\left(\begin{array}{cc}\gamma_{\ell} & q_{\ell}^{T} \\ q_{\ell} & Q_{\ell}\end{array}\right)$. Then, $g_{\ell}(x)=\left\langle P_{\ell},\left(\begin{array}{cc}1 & x^{T} \\ x & x x^{T}\end{array}\right)\right\rangle$. Therefore, the following set $\hat{F}$ is a natural semidefinite relaxation of $F$ :

$$
\hat{F}:=\left\{x \in \mathbb{R}^{n} \mid\left(\begin{array}{l}
1 \\
x
\end{array}\right)=Y e_{0} \text { for some } Y \succeq 0 \text { with }\left\langle P_{\ell}, Y\right\rangle \geq 0 \text { for } \ell=1, \ldots, m\right\}
$$

(considered, e.g., in (Fujie and Kojima 1997)). In fact, the set $\hat{F}$ coincides with the first Lasserre relaxation $\mathcal{Q}_{0}(F)$.

Proposition 25. $\mathcal{Q}_{0}(F)=\hat{F}$.

Proof. By definition, $x \in \mathbb{R}^{n}$ belongs to $\mathcal{Q}_{0}(F)$ if there exists $y=\left(y_{\alpha}\right)_{|\alpha| \leq 2}$ satisfying $y_{0}=1, y_{e_{i}}=x_{i}$ $(i=1, \ldots, n)\left(e_{1}, \ldots, e_{n}\right.$ denoting the standard unit vectors in $\left.\mathbb{R}^{n}\right), M_{1}^{\mathbb{Z}}(y) \succeq 0$ and $g_{\ell} * y(0) \geq 0$ $(\ell=1, \ldots, m)$. The equality $\mathcal{Q}_{0}(F)=\hat{F}$ follows from the following fact: Given a symmetric matrix $Y=\left(Y_{i j}\right)_{i, j=0}^{n}$, define $y=\left(y_{\alpha}\right)_{|\alpha| \leq 2}$ by $y_{0}:=Y_{00}, y_{e_{i}}:=Y_{0 i}, y_{e_{i}+e_{j}}:=Y_{i j}(i, j=1, \ldots, n)$; then, $M_{1}(y)=Y$ and $g_{\ell} * y(0)=\left\langle P_{\ell}, Y\right\rangle$. 
Acknowledgements. The author thanks the two referees for their careful reading and useful suggestions that helped improve the presentation of the paper.

\section{References}

Anjos, M.F., Wolkowicz, H. 2002. Strengthened semidefinite relaxations via a second lifting for the max-cut problem. Discrete Applied Mathematics 119 79-106.

Balas, E., Ceria, S., Cornuéjols, G. 1993. A lift-and-project cutting plane algorithm for mixed 0 - 1 programs. Mathematical Programming 58 295-324.

Barahona, F., Mahjoub, A.R. 1986. On the cut polytope. Mathematical Programming 36 157-173.

Berg, C., Christensen, J.P.R., Jensen, C.U. 1979. A remark on the multidimensional moment problem. Mathematische Annalen 243 163-169.

Berg, C., Christensen, J.P.R., Ressel, P. 1984. Harmonic Analysis and Semigroups - Theory of Positive Definite and Related Functions. Springer-Verlag, New York.

Chvátal, V. 1973. Edmonds polytopes and a hierarchy of combinatorial problems. Discrete Mathematics 4 305-337.

Chvátal, V., Cook, W., Hartman, M. 1989. On cutting-plane proofs in combinatorial optimization. Linear Algebra and its Applications 114/115 455-499.

Cook, W., Dash, S. 2001. On the matrix-cut rank of polyhedra. Mathematics of Operations Research 26 19-30.

Cornuéjols, G., Li, Y. 2001. On the rank of mixed 0-1 polyhedra. In K. Aardal and A.M.H. Gerards, eds., IPCO 2001, Lecture Notes in Computer Science 2081 71-77.

Curto, R.E., Fialkow, L.A. 2000. The truncated complex $K$-moment problem. Transactions of the American Mathematical Society 352 2825-2855.

Eisenbrand, F. 2000. On the membership problem for the elementary closure of a polyhedron. Combinatorica 19 299-300.

Eisenbrand, F., Schulz, A.S. 1999. Bounds on the Chvátal rank of polytopes in the 0/1 cube. In G. Cornuéjols, R.E. Burkard and G.J. Woeginger, eds., IPCO 1999, Lecture Notes in Computer Science $1610137-150$.

Fuglede, B. 1983. The multidimensional moment problem. Expositiones Mathematicae 1 47-65.

Fujie, T., Kojima, M. 1997. Semidefinite programming relaxation for nonconvex quadratic programs. Journal of Global Optimization 10 367-380.

Goemans, M.X., Tunçel, L. 2001. When does the positive semidefiniteness constraint help in lifting procedures. Mathematics of Operations Research 26 796-815.

Goemans, M.X., Williamson, D.P. 1995. Improved approximation algorithms for maximum cuts and satisfiability problems using semidefinite programming. Journal of the Association for Computing Machinery 42 1115-1145.

Grötschel, M., Lovász, L., Schrijver, A. 1988. Geometric Algorithms and Combinatorial Optimization. Springer Verlag, Berlin, New York.

Handelman, D. 1988. Representing polynomials by positive linear functions on compact convex polyhedra. Pacific Journal of Mathematics 132 35-62.

Haviland, E.K. 1935. On the momentum problem for distributions in more than one dimension. I. II. American Journal of Mathematics 57 562-568. 
Haviland, E.K. 1936. On the momentum problem for distributions in more than one dimension. I. II. American Journal of Mathematics 58 164-168.

De Klerk, E., Pasechnik, D.V. 2002. Approximating the stability number of a graph via copositive programming. SIAM Journal on Optimization 12 875-892.

Lasserre, J.B. 2000. Optimality conditions and LMI relaxations for $0-1$ programs. Technical Report N. 00099, CNRS-LAAS, Toulouse.

Lasserre, J.B. 2001a. Global optimization with polynomials and the problem of moments. SIAM Journal on Optimization 11 796-817.

Lasserre, J.B. 2001b. An explicit exact SDP relaxation for nonlinear 0-1 programs. In K. Aardal and A.M.H. Gerards, eds., Lecture Notes in Computer Science 2081 293-303.

Lasserre, J.B. 2002. Semidefinite programming vs. LP relaxations for polynomial programming. Mathematics of Operations Research 27 347-360.

Laurent, M. 2001. Tighter linear and semidefinite relaxations for max-cut based on the LovászSchrijver lift-and-project procedure. SIAM Journal on Optimization 12 345-375.

Laurent, M. 2002. Semidefinite relaxations for Max-Cut. To appear in The Sharpest Cut, Festschrift in Honor of M. Padberg's 60th Birthday, M. Grötschel, ed., SIAM.

Lovász, L. 1979. On the Shannon capacity of a graph. IEEE Transactions on Information Theory IT-25 $1-7$.

Lovász, L., Schrijver, A. 1991. Cones of matrices and set-functions and $0-1$ optimization. SIAM Journal on Optimization 1 166-190.

Nesterov, Y. 2000. Squared functional systems and optimization problems. In J.B.G. Frenk, C. Roos, T. Terlaky, and S. Zhang, eds., High Performance Optimization, pages 405-440. Kluwer Academic Publishers.

Parrilo, P.A. 2000. Structured semidefinite programs and semialgebraic geometry methods in robustness and optimization. PhD thesis, California Institute of Technology.

Putinar, M. 1993. Positive polynomials on compact semi-algebraic sets. Indiana University Mathematics Journal 42 969-984.

Reznick, B. 1998. Some concrete aspects of Hilbert's 17th problem. Report 98-002, Department of Mathematics, University of Illinois at Urbana-Champaign.

Schmüdgen, K. 1991. The K-moment problem for compact semi-algebraic sets. Mathematische Annalen 289 203-206.

Sherali, H.D., Adams, W.P. 1990. A hierarchy of relaxations between the continuous and convex hull representations for zero-one programming problems. SIAM Journal on Discrete Mathematics 3 411-430.

Sherali, H.D., Adams, W.P. 1994. A hierarchy of relaxations and convex hull characterizations for mixed-integer zero-one programming problems. Discrete and Applied Mathematics 52 83-106.

Sherali, H.D., Adams, W.P. 1999. A Reformulation-Linearization Technique for Solving Discrete and Continuous Nonconvex Problems. Kluwer, Boston, MA.

Sherali, H.D., Adams, W.P., Driscoll, P.J. 1998. Exploiting special structures in constructing a hierarchy of relaxations for 0-1 mixed integer problems. Operations Research 46 396-405.

Sherali, H.D., Tuncbilek, C.H. 1992. A global optimization algorithm for polynomial programming problems using a Reformulation-Linearization Technique. Journal of Global Optimization 2 101-112. 
Sherali, H.D., Tuncbilek, C.H. 1997. Reformulation-Linearization/Convexification relaxations for univariate and multivariate polynomial programming problems. Operations Research Letters 21 1-10.

Shor, N.Z. 1987. An approach to obtaining global extremums in polynomial mathematical programming problems. Kibernetika 5 102-106.

Shor, N.Z. 1998. Nondifferentiable Optimization and Polynomial Problems. Kluwer Academic Publishers.

Stephen, T., Tunçel, L. 1999. On a representation of the matching polytope via semidefinite liftings. Mathematics of Operations Research 24 1-7.

Wilf, H.S. 1968. Hadamard determinants, Möbius functions, and the chromatic number of a graph. Bulletin of the American Mathmatical Society 74 960-964. 